\title{
Specificity of bispecific T cell receptors and antibodies targeting peptide-HLA
}

\author{
Christopher J. Holland, ${ }^{1}$ Rory M. Crean, ${ }^{2,3}$ Johanne M. Pentier, ${ }^{1}$ Ben de Wet, ${ }^{1}$ Angharad Lloyd, ${ }^{1}$ Velupillai Srikannathasan, ${ }^{1}$ \\ Nikolai Lissin, ${ }^{1}$ Katy A. Lloyd, ${ }^{1}$ Thomas H. Blicher, ${ }^{1}$ Paul J. Conroy, ${ }^{1}$ Miriam Hock, ${ }^{1}$ Robert J. Pengelly, ${ }^{1}$ Thomas E. Spinner, \\ Brian Cameron, ${ }^{1}$ Elizabeth A. Potter, ${ }^{1}$ Anitha Jeyanthan, ${ }^{1}$ Peter E. Molloy, ${ }^{1}$ Malkit Sami, ${ }^{1}$ Milos Aleksic, ${ }^{1}$ Nathaniel Liddy, ${ }^{1}$ \\ Ross A. Robinson, ${ }^{1}$ Stephen Harper, ${ }^{1}$ Marco Lepore, ${ }^{1}$ Chris R. Pudney, ${ }^{2}$ Marc W. van der Kamp, ${ }^{4}$ Pierre J. Rizkallah, ${ }^{5}$ \\ Bent K. Jakobsen, ${ }^{1}$ Annelise Vuidepot, ${ }^{1}$ and David K. Cole ${ }^{1,5}$
}

IImmunocore Ltd., Milton Park, Abingdon, United Kingdom. '2Department of Biology and Biochemistry and ${ }^{3}$ Doctoral Training Centre in Sustainable Chemical Technologies, University of Bath, Bath, United Kingdom. ${ }^{4}$ School of Biochemistry, University of Bristol, Bristol, United Kingdom. ${ }^{5}$ Cardiff University School of Medicine, Heath Park, Cardiff, United Kingdom.

\begin{abstract}
Tumor-associated peptide-human leukocyte antigen complexes (pHLAs) represent the largest pool of cell surface-expressed cancer-specific epitopes, making them attractive targets for cancer therapies. Soluble bispecific molecules that incorporate an anti-CD3 effector function are being developed to redirect T cells against these targets using 2 different approaches. The first achieves pHLA recognition via affinity-enhanced versions of natural TCRs (e.g., immune-mobilizing monoclonal T cell receptors against cancer [ImmTAC] molecules), whereas the second harnesses an antibody-based format (TCR-mimic antibodies). For both classes of reagent, target specificity is vital, considering the vast universe of potential pHLA molecules that can be presented on healthy cells. Here, we made use of structural, biochemical, and computational approaches to investigate the molecular rules underpinning the reactivity patterns of pHLA-targeting bispecifics. We demonstrate that affinity-enhanced TCRs engage pHLA using a comparatively broad and balanced energetic footprint, with interactions distributed over several HLA and peptide side chains. As ImmTAC molecules, these TCRs also retained a greater degree of pHLA selectivity, with less off-target activity in cellular assays. Conversely, TCR-mimic antibodies tended to exhibit binding modes focused more toward hot spots on the HLA surface and exhibited a greater degree of crossreactivity. Our findings extend our understanding of the basic principles that underpin pHLA selectivity and exemplify a number of molecular approaches that can be used to probe the specificity of pHLA-targeting molecules, aiding the development of future reagents.
\end{abstract}

\section{Introduction}

The ability to selectively target tumor-specific antigens holds great promise for the development of specific cancer treatments, but their identification remains a key challenge. Peptide fragments presented on the cell surface by human leukocyte antigens (pHLAs) represent the intracellular proteome, and because this also includes dysregulated and cancerspecific proteins $(1,2)$, pHLAs constitute an important source of tumor-specific antigens. However, targeting these molecules is difficult for 2 reasons. First, their natural presentation levels can be very low (often below 10 copies of each specific peptide epitope per cell) (3); and second, peptides are corecognized in the context of HLA, a molecule expressed by most cells (i.e., peptide selectivity could be lost if HLA interactions dominate the binding interface) $(4,5)$.

Authorship note: $\mathrm{CJ} H, \mathrm{RMC}, \mathrm{JMP}, \mathrm{BDW}$, and $\mathrm{AL}$ contributed equally to this study. Conflict of interest: CJH, JMP, BDW, AL, N. Lissin, KAL, THB, SH, PJC, EAP, MH, RJP, TES, BC, AJ, PEM, AV, MS, MA, N. Liddy, RAR, SH, VS, ML, BKJ, and DKC are employees of Immunocore LTD

Copyright: @ 2020, American Society for Clinical Investigation.

Submitted: May 23, 2019; Accepted: February 11, 2020; Published: April 20, 2020.

Reference information: J Clin Invest. 2020;130(5):2673-2688.

https://doi.org/10.1172/JCI130562.
The immune system naturally overcomes these hurdles via selective $\mathrm{T}$ cell receptor (TCR) recognition of $\mathrm{pHLA}$, enabling $\mathrm{T}$ cell triggering toward low-level antigens (6-8). Although the mechanisms that determine peptide selectivity by natural TCRs are not fully understood, the binding mode employed by the TCR is likely to be fundamentally important, as evidenced by the conserved binding mode observed for virtually all TCR-pHLA structures solved to date (9). This canonical interaction places the TCR diagonally across the HLA-binding groove, positioning the somatically rearranged TCR complementarity determining region 3 (CDR3) loops centrally over the antigenic determinant (peptide) with the germline-encoded CDR1/2 loops positioned primarily over the HLA helices, enabling natural TCRs to detect pHLA in a peptidedependent manner. Despite the need for precise peptide selectivity, a limited number of TCRs must still maintain the ability to recognize millions of potential target antigens $(10,11)$. Consequently, TCRs have been shown to crossreact with a vast array of different peptides (11-14), but are selected in the thymus to avoid having specificities overlapping with abundant self-epitopes to maintain self-tolerance. Although the mechanisms that underpin these characteristics have yet to be determined, the relatively weak binding affinity of thymically selected TCRs ( $K_{D} \mathrm{~s}$ in the micromolar affinity range; refs. 15, 16) has been shown to be important for T cell sensitivity (17) and is likely also important for maintaining self-tolerance. 
The weak affinity of naturally selected TCRs, combined with difficulties manufacturing a membrane-bound protein as a soluble reagent, imposes certain challenges on their use for therapeutic applications. Consequently, the most widely used $\mathrm{T}$ cell-based therapies involve the adoptive transfer of either expanded antigen-specific $\mathrm{T}$ cells or $\mathrm{T}$ cells genetically modified to express an artificial antigen-specific TCR (specific peptide affinity-enhanced receptor [SPEAR]) (18) or antibody (chimeric antigen receptor [CAR]) (19). Although promising, these therapies are complicated by the need to prepare therapeutic $\mathrm{T}$ cells on a patient-by-patient basis and an inability to control dosing in response to potential toxicities (20).

Soluble bispecific $\mathrm{T}$ cell redirectors, consisting of antigen recognition and $\mathrm{T}$ cell-engaging domains, bypass many of the limitations of the adoptive transfer approach (21). The antigen recognition of pHLA-targeting reagents may be via a TCR or antibody domain. Immune-mobilizing monoclonal $\mathrm{T}$ cell receptors against cancer (ImmTAC) molecules are bispecific molecules with an engineered soluble TCR fused to an anti-CD3 effector function (22); thus, these molecules redirect T cells specifically toward cells presenting a target pHLA (22). The TCR component of ImmTAC molecules is stabilized with an interchain disulphide bond (23) and affinity enhanced using phage display to generate highly stable, soluble TCR reagents that can bind to pHLA with low-picomolar affinities and with binding half-lives of several hours (in comparison with half-lives of seconds for WT soluble TCRs) $(22,24)$. These attributes enable ImmTAC molecules to elicit antitumor responses at picomolar concentrations against cells expressing very low levels of pHLA on the cell surface. In comparison, bispecific $\mathrm{T}$ cell engagers (BiTEs) can utilize antibodies to target pHLA (TCR-mimic antibodies) as soluble $\mathrm{T}$ cell-engaging bispecific molecules (25-38). Antibodies, unlike TCRs that are anchored in the cell membrane, can exist naturally as soluble effector molecules (and as such, are easier to engineer as soluble reagents) and typically have a strong affinity for their antigen (nanomolar range), making them attractive for development as soluble therapeutics (21). The main challenge for a targeted pHLA therapeutic is achieving sufficient specificity in the context of a vast landscape of potential self-antigens. For instance, even on individual cell types, data from our in-house mass spectrometry database and published direct evidence demonstrate that the number of unique peptides can be in the range of tens of thousands (39-42). Considering the full human protein-coding genome, the number of peptides presented has been estimated to be over 11 million (43).

In this study, we used a combination of structural, molecular, and computational approaches to understand the molecular mechanisms underpinning pHLA selectivity and, consequently, the potential crossreactivity of soluble bispecific T cell redirectors. We demonstrate that utilization of a native TCR-like binding mode was not predictive of peptide selectivity. In fact, peptide selectivity, as defined by lower levels of pHLA crossreactivity and less offtarget activity in cellular testing, was associated with an energetic signature characterized by broad interactions with several peptide side chains as well as the peptide backbone. These findings have important implications for the underlying rules that determine pHLA discrimination and identify key considerations in the design of immunotherapeutics that target these cell-surface proteins.

\section{Results}

Structural analysis of pHLA-targeting reagents. We selected TCR-mimic antibodies according to in vitro and in vivo testing and based on the availability of crystal complex structures to enable molecular analysis (Supplemental Table 1; supplemental material available online with this article; https://doi.org/10.1172/ JCI130562DS1). Several additional TCR mimics have been reported; however, most lacked sufficient published information for inclusion (sequence, structure, and specificity data). As the peptide antigen can have a major influence on specificity (i.e., some peptides may have close homology to self-peptides), we chose affinity-enhanced TCRs based on their recognition of identical, or closely related, pHLA determinants compared with the TCR mimics. Here, we assessed reagents designed to recognize the NY-ESO- $1_{157-165}$ cancer testis antigen-derived peptide SLLMWITQC, presented by HLA-A*02:01 (A2-SLL), the MAGE-A1 ${ }_{161-169} /$ MAGE3-A3 ${ }_{168-176}$ melanoma-associated antigen-derived peptides EADPTGHSY (A1-EAD) and EVDPIGHLY (A1-EVD), presented by HLA-A ${ }^{*} 01: 01$, and the $\mathrm{WT}_{26-134}$ Wilms tumor antigen-derived peptide RMFPNAPYL, presented by HLA-A*02:01 (A2-RMF). Although not a direct comparison, the A1-EAD and A1-EVD still represented a useful system to include due to the similar tumorexpression patterns of both proteins, the same HLA restriction, and similar peptide sequences - a consequence of both peptides representing the same region of the highly related MAGE proteins.

In addition to the previously published crystal structures for TCR-mimic antibodies and affinity-enhanced TCRs in complex with A2-SLL $(36,44,45)$, A2-RMF (25), and A1-EVD/A1-EAD (refs. 46, 47, and Figure 1A), we solved the structure of the affinityenhanced WT1_ $\alpha_{7} \beta_{2}$ TCR in complex with A2-RMF at $2.8 \AA$ to complete the set (Table 1). Together, these data were analyzed to identify any structural features that might influence the peptide selectivity of each reagent. We compared the normal range of binding (crossing angle and engagement zone) of natural TCRs (9) with both affinity-enhanced TCRs and TCR-mimic antibodies. The affinity-enhanced TCRs $\left(1 G 44_{-} \alpha_{58} \beta_{61}\right.$ TCR, MAG-IC3 TCR, and WT1_ $\alpha_{7} \beta_{2}$ TCR) bound within the normal range of natural TCR topologies, with the CDR3 loops of both chains focused over the central peptide bulge (amino acids 4-6) (Figure 1B). This binding mode enabled the affinity-enhanced TCRs to form contacts with 5 of the 9 amino acids in the peptide and multiple interactions with the $\alpha_{1}$ and $\alpha_{2}$ domains of the HLA (Table 2). The $1 G 4{ }_{-} \alpha_{58} \beta_{61}$ and WT1_ $\alpha_{7} \beta_{2}$ TCRs were more peptide focused than the MAG-IC3 TCRs in terms of the percentages (57\%, 36\%, and $14 \%$, respectively) and numbers $(115,64$, and 16 , respectively) of peptide bonds. The $1 \mathrm{G} 4{ }_{-} \alpha_{58} \beta_{61}$ and WT1 $\alpha_{7} \beta_{2}$ TCRs also exhibited larger buried surface areas (BSAs) than MAG-IC3, but all were within, or near to, the normal range (1240-2400 $\AA^{2}$ ) (9). The 3M4E5 TCR-mimic antibody bound in a fashion very similar to that of natural TCRs, making contacts with 5 peptide residues, $38 \%$ peptide contacts, and a BSA of $2502 \AA^{2}$. However, the structural analysis revealed focusing of interactions at peptide residue W5, where half of the peptide contacts were concentrated $(24 / 51)$. Although the $1 G 4{ }_{-} \alpha_{58} \beta_{61}$ TCR also made many contacts with the large exposed side chain of W5 (54/115), binding was less focused on this residue and additional important contacts were made with other peptide residues, particularly M4 (31/115) and Q8 (15/115). 


\section{A}
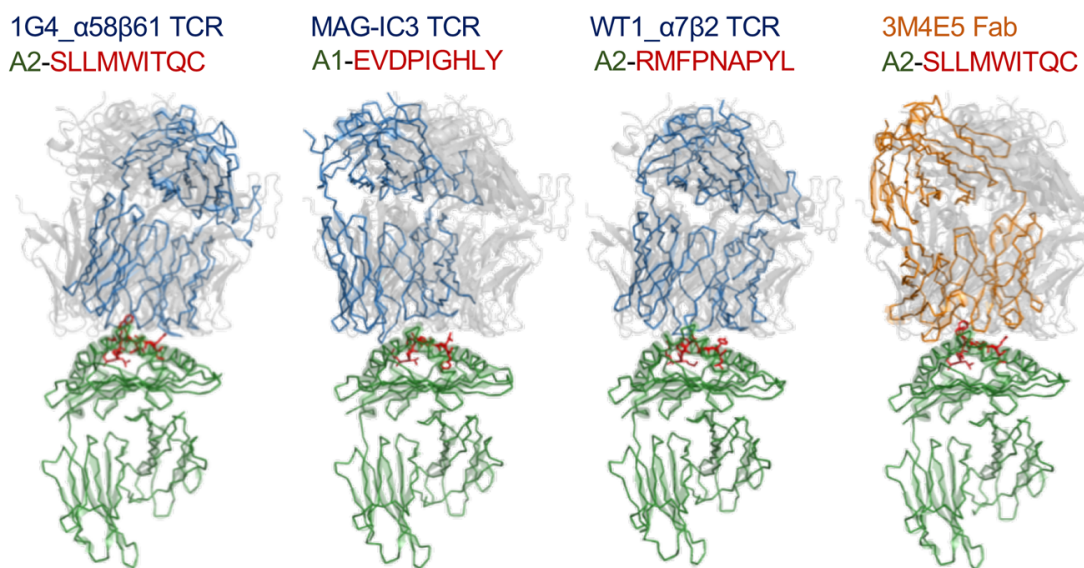

Hyb3.3 Fab A1-EADPTGHSY

ESK-1 Fab


A2-RMFPNAPYL
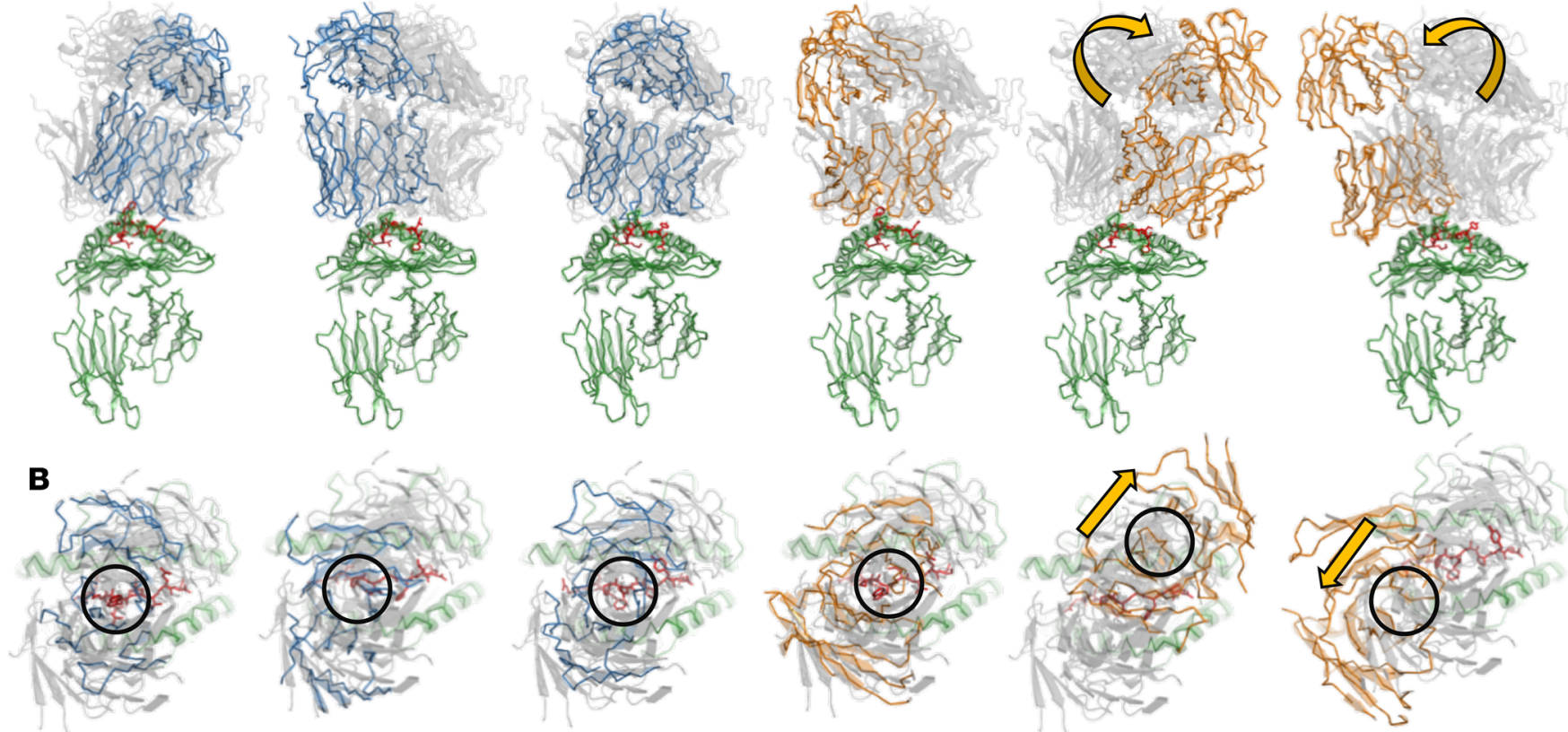

Figure 1. Structural analysis of pHLA-targeting reagents. Structures of the TCRs and TCR mimics, in complex with pHLA, were analyzed to determine the structural mechanism underpinning their binding characteristics. (A) TCRs (blue ribbon) or TCR mimics (orange ribbon) binding to peptide (red sticks) and HLA (green ribbon) compared with the binding range employed by all published WT TCR structures (gray drawing). Yellow arrows indicate unconventional binding modes. (B) Colors are as in A. Top down view of TCR or TCR mimic binding to pHLA. Black circles represent the center of binding. Yellow arrows indicate unconventional binding modes.

Peptide-binding hot spots have been detected for natural TCRs $(12,48,49)$; however, this structural feature has been associated with autoreactive TCRs and can correlate with a high level of TCR crossreactivity $(14,49)$. In contrast, the Hyb3.3 and ESK-1-mimic antibodies both bound to their respective pHLA using unconventional topology. Hyb3.3 binding was C-terminally shifted with a crossing angle substantially outside of the natural TCR-pHLA range. Despite this unusual topology, Hyb3.3 retained broad peptide contacts across 6 of the 9 amino acids, making $25 \%$ peptide contacts and a BSA of $2024 \AA^{2}$ (Table 2). ESK-1 binding was Nterminally shifted with an unconventional crossing angle. This binding mode positioned the TCR-mimic antibody so that the CDR3 loops were focused over the $\alpha_{2}$ helix of the HLA, resulting in a very limited interaction with the peptide. This binding mode resulted in ESK-1 making only $10 \%$ peptide contacts, and the majority of these were formed with peptide residue 1 . This HLA-centric binding mode, where only 1 peptide residue was contacted, raised questions about the ability of the ESK-1 TCR mimic to retain specificity. However, for all the other HLA-targeting reagents considered here, their structures appeared to retain many of the features observed for natural TCR-pHLA interactions.

Alanine scan analysis reveals distinct molecular recognition patterns between TCRs and TCR-mimic antibodies. Alanine scan mutagenesis was used to investigate the molecular recognition pattern of the affinity-enhanced TCRs and TCR-mimic antibodies using surface plasmon resonance (Figure 2 and Supplemental Figure 1). For each reagent, we generated a panel of soluble pHLAs in which each peptide residue was replaced with an alanine (or a serine if the native residue was already an alanine), except for the canonical anchor residues at positions 2 and 9. The 1G4_ $\alpha_{58} \beta_{61}$ TCR bound to A2-SLL with a $K_{D}=57 \mathrm{pM}$. However, binding was not detected when residues 5 and 6 were mutated to alanine, and the affinity was substantially reduced when residues 4,7 , and 8 were mutated (Figure 2A). These findings are consistent with the $1 G 4 \alpha_{-} \alpha_{58} \beta_{61}-$ A2-SLL cocomplex crystal structure showing that the central MW motif forms a central peptide bulge, making multiple contacts with the TCR CDR3 loops, and peptide residue Q8 points up away from the HLA surface, enabling contacts with the TCR CDR1 $\beta$ loop (Figure 1 and Table 2). A similar pattern was observed for the $1 \mathrm{G} 4{ }_{-} \alpha_{5} \beta 5_{1} \mathrm{~A} 2$-SLL restricted TCR (a different affinity mutant version of $1 G 4 \alpha_{58} \beta_{61}$ originating from the same progenitor WT TCR, $\mathrm{K}_{\mathrm{D}}=1.4 \mathrm{nM}$ ), with reductions in affinity additionally observed at peptide positions 1 and 3, whereas the $1 \mathrm{G} 4_{-} \alpha_{5} \beta_{100}$ A2-SLL restricted TCR (a different affinity mutant version of $1 G 44_{-} \alpha_{58} \beta_{61}$ originating from the same progenitor WT TCR), which bound with a weaker affinity $\left(K_{D}=5 \mathrm{nM}\right)$, was highly sensitive to alanine mutations at every position along the peptide backbone (Figure $2 \mathrm{~A}$ ). We repeated the alanine scan analysis on the A2-SLLreactive 3M4E5 TCR mimic and included 2 published higher affinity versions of 3M4E5 (36) (3M4E5_T2 and 3M4E5_T3) because they were closer in affinity to the $1 \mathrm{G} 4_{-} \alpha_{5} \beta_{100}$ and $1 \mathrm{G} 4_{-} \alpha_{5} \beta_{51}$ affinityenhanced TCRs, allowing a more direct comparison. The 3M4E5 $\left(K_{D}=44 \mathrm{nM}\right.$ in single-chain fusion [scFv] format) and 3M4E5_T2 TCR-mimic antibodies $\left(\mathrm{K}_{\mathrm{D}}=2.8 \mathrm{nM}\right.$ in scFv format) were both 
Table 1. Data collection and refinement statistics for WT1_ $\alpha_{7} \beta_{2}-$ A2-RMF and NYBR1-A2-SLS structures

\begin{tabular}{|c|c|c|}
\hline & WT1_ $\boldsymbol{\alpha}_{7} \boldsymbol{\beta}_{2}$-A2-RMF & NYBR1-A2-SLS \\
\hline PDB code & 6RSY & 6R2L \\
\hline \multicolumn{3}{|l|}{${ }^{A}$ Data collection } \\
\hline Space group & P 212121 & P1211 \\
\hline \multicolumn{3}{|l|}{ Cell dimensions } \\
\hline a, b, c ( $(\AA)$ & $94.6,114.8,185.4$ & $52.2,99.3,111.4$ \\
\hline$\alpha, \beta, \gamma\left(^{\circ}\right)$ & $90,90,90$ & $90,90.5,90$ \\
\hline Resolution (A) & $114.8-.03^{\mathrm{B}}(3.0-3.0)$ & $52.2-2.4^{B}(2.4-2.3)$ \\
\hline Beam line & $104-1$ & 104-1 \\
\hline Beam time code & In17077-18 & $14843-1$ \\
\hline $\mathrm{R}_{\text {merge }}(\%)$ & $32.6^{\mathrm{B}}(220.4)$ & $20.7^{\mathrm{B}}(108.9)$ \\
\hline$|/ \sigma|$ & $8.8^{B}(1.2)$ & $9.7^{B}(1.3)$ \\
\hline Completeness (\%) & $99.9^{\mathrm{B}}(99.9)$ & $99.7^{\mathrm{B}}(99.7)$ \\
\hline Redundancy & $14.1^{\mathrm{B}}(14.3)$ & $3.7^{\mathrm{B}}(3.6)$ \\
\hline No. reflections & $700,229^{\mathrm{B}}(54,486)$ & $188,042^{\mathrm{B}}(13,553)$ \\
\hline \multicolumn{3}{|l|}{ Refinement } \\
\hline No $R_{\text {free }}$ reflections & 2,370 & 2,435 \\
\hline $\mathrm{R}_{\text {work }} / \mathrm{R}_{\text {free }}$ & 25.1/29.1 & $21.4 / 26.5$ \\
\hline \multicolumn{3}{|l|}{ R.m.s. deviations } \\
\hline Bond lengths ( $(\AA)$ & 0.006 & 0.011 \\
\hline Bond Angles $\left({ }^{\circ}\right)$ & 1.253 & 1.853 \\
\hline Mean $B$ value $\left(\AA^{2}\right)$ & 75.5 & 40.0 \\
\hline Wilson B factor $\left(\AA^{2}\right)$ & 75.8 & 25.9 \\
\hline $\begin{array}{l}\text { Estimated coordinate error based } \\
\text { on maximum likelihood }(A)\end{array}$ & 0.423 & 0.221 \\
\hline
\end{tabular}

${ }^{A}$ One crystal was used for data collection and solving each structure.

${ }^{B}$ Figures in parentheses refer to the highest resolution bin.

sensitive to alanine mutation at peptide residues 4,5 , and 6 (Figure $2 \mathrm{~B}$ ), whereas mutations at all other positions of the peptide did not reduce binding affinity. $3 \mathrm{M} 4 \mathrm{E} 5 \mathrm{~T} 33\left(K_{\mathrm{D}}=5.5 \mathrm{nM}\right.$ in scFv format) demonstrated a similar trend, being sensitive to alanine substitution at peptide residues 4 and 5 (Figure 2B). Alanine substitutions at peptide residues $1,3,7$, and 8 had no impact on binding affinity for any of the A2-SLL TCR mimics, demonstrating a more focused binding mode around peptide residues 4,5 , and 6 compared with the affinity-enhanced TCRs. These findings were also consistent with the crystal structure of 3M4E5-A2-SLL that demonstrated binding was focused toward these central residues of the peptide.

The high level of sensitivity to alanine substitutions across the peptide backbone was also observed for the A1-EVD-specific MAG-IC3 $\left(K_{D}=3.8 \mathrm{nM}\right)$ and MAG-IC5 (a different affinity mutant version of MAG-IC3 TCR originating from the same progenitor WT TCR, $K_{D}=17 \mathrm{nM}$ ) TCRs (Figure 2C). The stronger affinity MAG-IC3 TCR demonstrated reduced or abrogated affinity toward every alanine mutant tested, while the MAG-IC5 TCR was sensitive to mutations at all positions apart from peptide residues 6 and 7. The MAG-IC3-A1-EVD cocomplex crystal structure was consistent with this finding, demonstrating a complex network of contacts across the peptide backbone (Figure 1 and Table 2). The Hyb3.3 TCR-mimic antibody recognizes the same peptide region as MAG-IC3 and MAG-IC5, but derived from a different MAGE protein (MAGE-A1), and binds with an affinity of $K_{D}=18 \mathrm{nM}$. The MAGE-A3 and MAGE-A1 peptides are conserved at all positions except for the N-terminal anchor (position 2), position 5 , and position 8. Although the Hyb3.3-A1-EAD complex structure demonstrated noncanonical topology, the interactions with the peptide apparent in the structure were comparable to most TCR-pHLA complexes (Figure 1 and Table 2). This observation was reflected in the alanine scan analysis, which demonstrated some degree of sensitivity to alanine substitutions at all positions tested apart from peptide residue 1 (Figure 2D).

The WT1_ $\alpha_{7} \beta_{2}$ TCR, which bound A2-RMF with a $K_{D}=70$ $\mathrm{nM}$, exhibited abrogated or highly reduced binding for all residues except at peptide position 6 . The WT1_ $\alpha_{27} \beta_{2}\left(K_{D}=13 \mathrm{nM}\right)$ and WT1_ $\alpha_{42} \beta_{2}\left(K_{D}=0.76 \mathrm{nM}\right)$ TCRs (both different affinity mutant versions of WT1_ $\alpha_{7} \beta_{2}$ TCR originating from the same progenitor WT TCR) showed a similar trend, with the strongest binding WT1_ $\alpha_{42} \beta_{2}$ TCR exhibiting the greatest level of sensitivity to alanine substitutions across the peptide backbone (Figure 2E). The ESK1 TCR-mimic antibody, which has a relatively weak affinity for A2-RMF of $K_{D}=2 \mu \mathrm{M}$ in scFv format (Supplemental Figure 1), demonstrated broad degeneracy in peptide binding, being tolerant to alanine substitutions at all positions of the peptide except for peptide residue 1 (Figure 2F). Here, availability of the ESK-1-A2-WT-1 structure provided insight into this observation, confirming that virtually all contacts between ESK-1 and the WT-1 peptide were focused on peptide residue 1 (Figure 1 and Table 2).

TCR-mimic antibodies bind to several commonly expressed self-peptides. Although alanine scan analysis is useful for understanding positional sensitivity of pHLA-targeting receptors, it is unclear how these data relate to the broader crossreactivity of these reagents, particularly their ability to discriminate against common self-peptides. To gain further insight into self-discrimination, we designed an experimental approach for screening multiple pHLA complexes in a high-throughput format by modifying the MagPix platform. We designed multiplex experiments using MagPlex beads coated with HLAs in complex with a range of commonly expressed self-peptides. Self-peptide HLAs recognized by affinity-enhanced TCRs or TCR mimics were detected using MagPix analysis (Tables 3 and 4). In all cases, the affinity-enhanced TCR reagents only generated a signal against their respective index peptides, whereas the TCR-mimic antibodies (in scFv format to avoid avidity-mediated binding) were more promiscuous. The 3M4E5_T2 scFv was reactive against 4 broadly expressed HLA-A ${ }^{*} 02: 01$ restricted self-peptides, and the ESK1 scFv demonstrated reactivity against 6 broadly expressed HLA-A ${ }^{*} 02: 01$ restricted self-peptides, in addition to their target antigens A2-SLL and A2-RMF, respectively (Table 3). The Hyb3.3 scFv was reactive against almost all of the HLA-A ${ }^{*} 01: 01$ restricted self-peptides tested (9/12) as well as A1-EAD (Table 4). In all cases, the selfpeptides recognized by the TCR-mimic antibodies shared very little sequence similarity, revealing a high level of potential crossreactivity compared with the affinity-enhanced TCRs developed to target identical, or very similar, peptide antigens.

Deep sequencing of peptides from randomized PHLA phage libraries demonstrates the binding degeneracy of pHLA-targeting reagents. Despite the ability of 3M4E5 to bind pHLA in a TCR- 
Table 2. Structural analyses of TCRs versus TCR mimics binding to pHLA

\begin{tabular}{|c|c|c|c|c|c|c|}
\hline & $1 \mathrm{CC}{ }_{-} \alpha_{58} \beta_{61} \mathrm{TCR}$ & MAG-IC3 TCR & WT1_ $\alpha_{7} \boldsymbol{\beta}_{2}$ TCR & 3M4E5 Fab & Hyb3.3 Fab & ESK-1 Fab \\
\hline Crossing angle $\left({ }^{\circ}\right)$ & 67 & 58.2 & 53.8 & 43.3 & 40 & 60.2 \\
\hline Pep bonds (bold >10) & $4, \underline{5}, 6,7,8$ & $1, \underline{4}, 5,7,8$ & $1,4,5,7, \underline{8}$ & $4, \underline{5}, 6,7,8$ & $4,5,6, \underline{7}, 8,9$ & 1,4 \\
\hline${ }^{B} \mathrm{HLA}>5$ bonds (bold $>10$ ) ${ }^{\mathrm{B}}$ & $\begin{array}{c}65,68,71, \\
72,73, \\
75,150,151,155\end{array}$ & $\begin{array}{l}66,154 \\
155,157 \\
158,163\end{array}$ & $\begin{array}{c}65,66,72 \\
75,150,151 \\
154,155\end{array}$ & $\begin{array}{c}65,66,72 \\
150,155 \\
158,163\end{array}$ & $\begin{array}{c}65,69,72 \\
73,76,80,82 \\
84,146,155\end{array}$ & $\begin{array}{c}55,56,58,59, \\
62,63,65,66, \\
155,162,167,169,170\end{array}$ \\
\hline APep bonds & $115(57 \%)$ & $16(14 \%)$ & $64(36 \%)$ & $51(38 \%)$ & $34(25 \%)$ & $19(10 \%)$ \\
\hline \multicolumn{7}{|l|}{$B S A\left(A^{2}\right)$} \\
\hline BSA total & 2422 & 2056 & 2640 & 2502 & 2024 & 2168 \\
\hline BSA $\alpha / \mathrm{H}$ Pep & 502 & 222 & 308 & 364 & 376 & 186 \\
\hline BSA $\beta /$ L Pep & 396 & 320 & 403 & 462 & 90 & 144 \\
\hline
\end{tabular}

ATotal bonds, HLA bond and Pep (peptide) bonds were assessed using a 3.4 Å cutoff for H-bonds and a 4 Å cutoff for vdW. ${ }^{B}$ Only HLA residues with more than 5 bonds are shown. Any peptide or HLA residue with more than 10 contacts is shown in bold. Peptide residue making the most contacts is underlined.

like conformation, the alanine scan profile and MagPix analysis revealed lower levels of peptide selectivity compared with the affinity-enhanced TCRs. To probe this discrepancy further, we developed an approach for the characterization of TCR peptide degeneracy using randomized pHLA libraries displayed on phage (50). We used this system to identify peptide motif preferences for the affinity-enhanced TCRs and TCR mimics that recognized
A2-SLL. In broad agreement with the structural and alanine scan analyses, the affinity-enhanced TCRs, $1 G 44_{-} \alpha_{5} \beta_{100}, 1 G 4{ }_{-} \alpha_{5} \beta_{51}$, and $1 G 4 \alpha_{58} \beta_{61}$, showed strong preferences for the native SLL peptide sequence at residues W5, T7, Q8, and V9 (Figure 3, A-C). In contrast, the TCR mimic 3M4E5 demonstrated a preference for the native SLL peptide sequence only at W5, with all other positions showing very little amino acid preference (Figure 3, D). Although

Table 3. MagPix analysis of commonly expressed HLA-A*0201-restricted self-peptides

\begin{tabular}{|c|c|c|c|c|c|c|c|}
\hline HLA & Antigen & Peptide & $1 \mathrm{C} 4{ }_{-} \boldsymbol{\alpha}_{5} \boldsymbol{\beta}_{100}$ TCR & $1 \mathrm{C} 4{ }_{-} \boldsymbol{\alpha}_{58} \boldsymbol{\beta}_{61}$ TCR & 3M4E5_T2 scFv & WT1_ $\alpha_{7} \beta_{2}$ TCR & ESK-1 scFv \\
\hline$A 2$ & SLLMWITQC & NY-ESO ${ }_{(157-165)}$ & 100.0 & 100.0 & 100.0 & 0.0 & 0.0 \\
\hline $\mathrm{A} 2$ & RMFPNAPYL & WT1 $_{(126-134)}$ & 0.0 & 0.0 & 0.6 & 100.0 & 100.0 \\
\hline$A 2$ & AIVDKVPSV & COPG1 $_{(147-155)}$ & 0.0 & 0.0 & 0.0 & 0.0 & 0.0 \\
\hline$A 2$ & ALVVQVAEA & $\operatorname{HEXB}_{(34-42)}$ & 0.0 & 0.0 & 0.0 & 0.0 & 0.1 \\
\hline$A 2$ & SLDQPTQTV & EIF3C $_{(834-842)}$ & 0.0 & 0.0 & 0.0 & 0.0 & 0.0 \\
\hline $\mathrm{A} 2$ & GLATDVQTV & $\operatorname{PSMB3}_{(55-63)}$ & 0.0 & 0.0 & 0.0 & 0.0 & 0.0 \\
\hline$A 2$ & ILTDITKGV & $\mathrm{EEF}_{(661-669)}$ & 0.0 & 0.0 & 0.0 & 0.0 & 0.0 \\
\hline $\mathrm{A} 2$ & IMLEALERV & SNRPGP15 $_{(68-76)}$ & 0.0 & 0.0 & 0.0 & 0.0 & 0.0 \\
\hline$A 2$ & VMDSKIVQV & KPNA1 $_{(434-442)}$ & 0.0 & 0.0 & 0.1 & 0.0 & 0.2 \\
\hline $\mathrm{A} 2$ & RLQEDPPAGV & UBE$A_{(15-24)}$ & 0.0 & 0.0 & 0.0 & 0.0 & 0.3 \\
\hline$A 2$ & KIYEGQVEV & $\mathrm{RPL}_{(117-125)}$ & 0.0 & 0.0 & 0.0 & 0.0 & 0.2 \\
\hline$A 2$ & NLAENISRV & $\operatorname{PYGM}_{(271-279)}$ & 0.0 & 0.0 & 0.0 & 0.0 & 0.0 \\
\hline$A 2$ & LLDVPTAAV & $\mathrm{IFI30} \mathrm{(16-24)}$ & 0.0 & 0.0 & 0.0 & 0.0 & 0.0 \\
\hline$A 2$ & SLSEKTVLL & $\operatorname{CD59}_{(106-114)}$ & 0.0 & 0.0 & 0.0 & 0.0 & 0.0 \\
\hline$A 2$ & ALNEKLVNL & EIF3F $_{(349-357)}$ & 0.0 & 0.0 & 0.0 & 0.0 & 0.0 \\
\hline$A 2$ & ILDKKVEKV & HSP9OAB1 $_{(570-578)}$ & 0.0 & 0.0 & 0.1 & 0.0 & 0.3 \\
\hline$A 2$ & ILDQKINEV & $\mathrm{ODC} 1_{(23-31)}$ & 0.0 & 0.0 & 0.0 & 0.0 & 0.0 \\
\hline$A 2$ & VLIDYQRNV & $\mathrm{XPO1}_{(784-792)}$ & 0.0 & 0.0 & 0.0 & 0.0 & 0.0 \\
\hline $\mathrm{A} 2$ & GLIEKNIEL & DNMT1 $_{(425-433)}$ & 0.0 & 0.0 & 0.0 & 0.0 & 0.0 \\
\hline$A 2$ & FLDPNNIPKA & $\operatorname{ALG8}_{(305-314)}$ & 0.0 & 0.0 & 0.0 & 0.0 & 0.0 \\
\hline$A 2$ & SLQSTILGV & LONP2 $_{(51-59)}$ & 0.0 & 0.0 & 0.2 & 0.0 & 0.0 \\
\hline $\mathrm{A} 2$ & SLYDYNPNL & EIF3F $_{(381-389)}$ & 0.0 & 0.0 & 0.0 & 0.0 & 0.0 \\
\hline
\end{tabular}


Table 4. MagPix analysis of commonly expressed HLA-A*0101-restricted selfpeptides

$\begin{array}{lcccc}\text { HLA } & \text { Antigen } & \text { Peptide } & \text { MAG-IC3 TCR } & \text { Hyb3.3 scFv } \\ \text { A1 } & \text { EVDPIGHLY } & \text { MAGE-A3 }_{(168-176)} & 100.0 & 70.0 \\ \text { A1 } & \text { EADPTCHSY } & \text { MAGE-A1 }_{(161-169)} & 0.0 & 100.0 \\ \text { A1 } & \text { YSDKYGLGY } & \text { PLK1 }_{(417-425)} & 0.0 & 90.7 \\ \text { A1 } & \text { DTDHYFLRY } & \text { PIGT }_{(165-173)} & 0.0 & 16.8 \\ \text { A1 } & \text { STDHIPILY } & \text { CFPT1 }_{(218-226)} & 0.0 & 0.4 \\ \text { A1 } & \text { HSDPSILGY } & \text { CIGYF1 }_{(1012-1020)} & 0.0 & 15.2 \\ \text { A1 } & \text { KSDVHLNFY } & \text { HLTF }_{(499-507)} & 0.0 & 0.0 \\ \text { A1 } & \text { HTDILKEKY } & \text { DTWD1 }_{(222-270)} & 0.0 & 0.0 \\ \text { A1 } & \text { IADMGHLKY } & \text { PCNA }_{(241-249)} & 0.0 & 1.0 \\ \text { A1 } & \text { LTELPDWSY }_{\text {A1 }} & \text { MRPL52 }_{(43-51)} & 0.0 & 0.0 \\ \text { A1 } & \text { ASDPFFRHY } & \text { CPN22 }_{(210-218)} & 0.0 & 77.3 \\ \text { A1 } & \text { ETEKDFSRY } & \text { AQR }_{(1757-895)} & 0.0 & 4.3\end{array}$

W5 was selected by $3 \mathrm{M} 4 \mathrm{E} 5$ _T2 and $3 \mathrm{M} 4 \mathrm{E} 5$-T3, it was not the dominant amino acid preference at this position, with phenylalanine (F) being preferred by both reagents (Figure 3, E-F). 3M4E5 T2 and 3M4E5_T3 also displayed very little selectivity in terms of amino acid preference at any other position. These findings are consistent with the alanine scan results demonstrating that the A2-SLL-reactive TCR mimics could tolerate alanine substitutions at any residue outside of the central MW peg in the SLL peptide, while the affinity-enhanced TCRs were selective across the peptide backbone.

Using this information, we assessed the number of unique peptides selected by each reagent to gain insight into their comparative crossreactivity. Analysis of next-generation sequencing data identified an average of 7068 unique peptides (687,241 total reads) for $1 G 44_{-} \alpha_{5} \beta_{51}, 4455$ unique peptides $(689,928$ total reads) for $1 G 4 \alpha_{5} \alpha_{5} \beta_{100}$, and 9012 unique peptides $(696,992$ total reads) for $1 G 4 \alpha_{58} \beta_{61}$. The TCR mimics selected between 3 and 15 times more unique peptides compared with the affinity-enhanced TCRs with 50,765 unique peptides (740,196 total reads) for 3M4E5, 60,699 unique $(692,455$ total reads) for 3M4E5_T2, and 32,934 unique (727,824 total reads) for 3M4E5_T3. Overall, these data suggest that the A2-SLL affinity-enhanced TCRs are less crossreactive (in terms of total number of peptides recognized) and less promiscuous in terms of their ability to tolerate amino acid variation across the peptide backbone compared with the A2-SLL TCR mimics.

Molecular dynamics simulations reveal peptide selectivity is associated with distinctive energetic modes of binding between TCRs and TCR-mimic antibodies. Although the structural and alanine scan analyses provided useful insights into the recognition mode employed by the pHLA-targeting molecules described here, they were not fully predictive of the recognition patterns observed in the MagPix and randomized pHLA library analysis. For example, despite the A2-SLL- and A1-EAD-reactive TCR mimics forming seemingly broad peptide contacts, according to the structural analysis, and promising alanine scan profiles, these reagents bound substantially more self-peptides in the MagPix analysis than the affinity-enhanced TCRs. Furthermore, the A2-SLL-reactive TCR mimics were characterized by more degenerate peptide binding in the randomized pHLA library analysis. Consequently, we performed molecular dynamics (MD) simulations to extend the "snapshot" view available from crystal structures. We subjected all 6 structures described in Figure 1 to two 500-nanosecond long MD simulations to investigate the biochemical nature and lifetime of contacts formed between the peptide and affinity-enhanced TCRs or TCR mimics. Interactions were dissected into contacts formed between the peptide side chain (amino acid specific) and main chain (conformation specific) versus time and separated into hydrogen bonding (H-bond) and van der Waals (vdW) type interactions. In all cases, the affinity-enhanced TCRs made a higher number of long-lived contacts with side-chain atoms across the peptide compared with main-chain interactions (Figure 4, A-C). In contrast, 3M4E5 made a lower number of peptide side-chain contacts (Figure $4 \mathrm{D})$, reflected by a lower overall ratio of peptide sidechain contacts (H-bond ratio: 0.5 , vdW ratio: 2.23 ) compared with the $1 G 4{ }_{-} \alpha_{58} \beta_{61}$ TCR (H-bond ratio: 1.49 , vdW ratio: 5.41 ) (Supplemental Figure 2). Hyb3.3 made virtually no contacts with peptide side-chain residues ( $\mathrm{H}$-bond ratio: 0.16 , vdW ratio: 0.24 ), focusing primarily or exclusively on interactions with the peptide backbone (Figure 4E and Supplemental Figure 2). ESK-1 did make peptide side-chain interactions, but only with the exposed R1, in line with the alanine scan analysis (Figure $4 \mathrm{~F}$ and Supplemental Figure 2). These data suggest that more limited side-chain-mediated recognition patterns, as observed for the TCR mimics, might contribute to greater levels of peptide degeneracy.

The energetic landscape of each affinity-enhanced TCR/ TCR mimic-pHLA complex was characterized by calculating their binding free energies (using the molecular mechanics PoissonBoltzmann surface area [MMPBSA] approach) (51). The MMPBSA approach has been used extensively to predict relative proteinligand and protein-protein binding free energies $(52,53)$. More specifically, it has been used to rationalize the effect of mutations on antibody-antigen complexes (54) and the role of a waterbridged interaction in TCR-pHLA affinity (55) and to predict reliable relative binding free energies of pHLA complexes (56). Here, we performed $25 \times 4$ nanosecond MD simulations per complex for MMPBSA analysis to ensure reliable and converged results (52, $56,57)$. MMPBSA calculations have the advantage that they can be decomposed into per residue contributions to the binding free energy, allowing one to predict each residue's preference toward binding. Analysis of the decomposition results demonstrated that the affinity-enhanced TCRs were characterized by broad energetic signatures, whereby the binding energy was distributed over at least 3 peptide residues, with multiple disparate regions driving affinity across the HLA surface (Figure 5, A-C, and Supplemental Figure 3, A-C). On the other hand, the TCR mimics were more HLA focused, with 1 or 2 energetic hot spots focused on single HLA residues (Figure 5, D-F, and Supplemental Figure 3, D-F). For example, although the $1 G 4{ }_{-} \alpha_{58} \beta_{61}$ TCR made substantial energetic interactions with HLA residue R65 $\left(-12 \mathrm{kcal} \mathrm{mol}^{-1}\right)$, this was bal- 

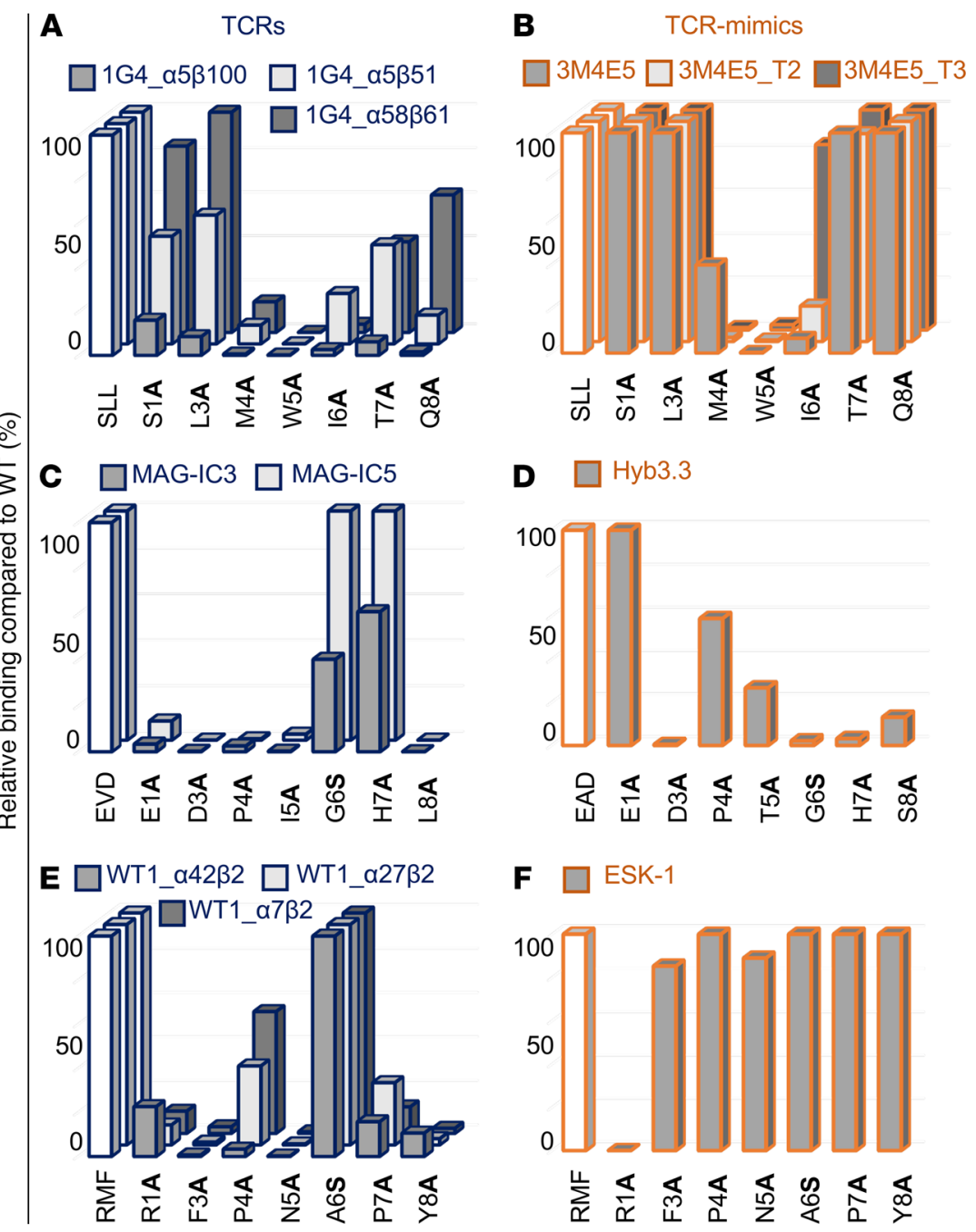

Figure 2. Alanine scan analysis reveals distinct molecular recognition patterns between TCRs and TCR-mimic antibodies. The contribution of peptide side chains to binding specificity was analyzed using alanine scan mutagenesis (by SPR). Binding affinities of the TCRs and TCR-mimic antibodies were determined using single-cycle kinetic analysis. Bar graphs show binding affinity as a percentage relative to the binding affinity to the index peptide. (A) A2-SLL affinity-enhanced TCRs, (B) A2-SLL TCR mimics, (C) A1-EVD affinity-enhanced TCRs, (D) Hyb3.3, (E) A2-RMF affinity-enhanced TCRs, and (F) ESK-1. Representative data from 3 independent experiments are shown.

anced by interactions with multiple peptide residues (W5:-11 kcal $\mathrm{mol}^{-1}$, M4:-8 $\mathrm{kcal} \mathrm{mol}^{-1}$, Q8:-7 $\left.\mathrm{kcal} \mathrm{mol}^{-1}\right)$ and other HLA residues (Q155:-6 kcal mol-1). The MAG-IC3 TCR had a balanced energetic footprint with important binding contributions from HLA residues E63, N66, and V158 as well as peptide residues E1, D3, P4, and $\mathrm{H} 7$ (all around $-5 \mathrm{kcal} \mathrm{mol}^{-1}$ ), and no energetic hot spots were detected for the WT1_ $\alpha_{7} \beta_{2}$ TCR, with most of the binding energy being equally balanced over HLA residues R65, R75, and Q155 and peptide residues $\mathrm{P} 4, \mathrm{~N} 5$, and $\mathrm{Y} 8$ (all around $-6 \mathrm{kcal} \mathrm{mol}^{-1}$ ). In contrast, all of the TCR mimics utilized more focused energetic binding to engage their cognate pHLAs. The chief energetic contribution for 3M4E5 was made by HLA residue R65 ( $-16 \mathrm{kcal}$ $\left.\mathrm{mol}^{-1}\right)$, with an additional peptide hot spot at W5 $\left(-7 \mathrm{kcal} \mathrm{mol}^{-1}\right)$. Hyb3.3 binding was characterized by strong energetically favorable interactions only with HLA residues K146 (-11 kcal mol-1) and R65 (-11 kcal mol-1), with the EAD peptide playing a minor role. Finally, for ESK-1, energetic hot spots were detected at HLA residue K66 (-15 $\left.\mathrm{kcal} \mathrm{mol}{ }^{-1}\right)$ and peptide residue $\mathrm{R} 1\left(-13 \mathrm{kcal} \mathrm{mol}^{-1}\right)$, with very little contribution from any other peptide residues.

Together with the previous analyses, the MD simulations provide further evidence that, rather than being driven by the recognition of a dominant amino acid at a single position on the HLA or peptide, broad interactions across the peptide (particularly with peptide side chains) were associated with greater peptide selectivity.

Redirected $T$ cell killing of antigen-positive and -negative cell lines using pHLA-targeting bispecifics. On-target versus off-target reactivity of affinity-enhanced TCRs and TCR mimics was assessed in functional $\mathrm{T}$ cell redirection assays against antigen-positive and antigen-negative cell lines. Cell lines were assessed for RNA transcript levels of the genes encoding each protein to determine their antigenic status (Supplemental Figure 4). Soluble bispecific molecules were generated by fusing an anti-CD3 scFv $\left(\mathrm{K}_{\mathrm{D}}=10\right.$ $\mathrm{nM}$, half-life $=2$ minutes) to the $\beta$ chain of the affinity-enhanced $\quad 1 G 4 \alpha_{58} \beta_{61} \quad\left(\right.$ IMC- $\left.1 G 4{ }_{-} \alpha_{58} \beta_{61}\right)$ and MAG-IC3 TCRs (IMC-MAG-IC3) or the heavy chain of the TCR mimic scFv 3M4E5_T2 (3M4E5_T2/anti-CD3), 3M4E5_T3 (3M4E5_T3/ anti-CD3), and Hyb3.3 (Hyb3.3/anti-CD3). Reagents recognizing A2-RMF were not included because, consistent with evidence from other studies (58), we were unable to detect the peptide on $\mathrm{WT}^{+}$tumor cells by mass spectrometry analysis (data not shown). IMC-1G4_ $\alpha_{58} \beta_{61}$ redirected $\mathrm{T}$ cell killing of $\mathrm{A2}^{+} \mathrm{NY}-\mathrm{ESO}^{+} \mathrm{NCIH}-1755$ cells was approximately 20 times more sensitive when compared with 3M4E5_T2/anti-CD3 and 3M4E5_T3/ anti-CD3, in line with the difference in affinity between these reagents (Figure 6A and Supplemental Figure 5). No redirected $\mathrm{T}$ cell killing of $\mathrm{A}^{+} \mathrm{NY}^{-\mathrm{ESO}^{-}}$targets was detected for IMC-1G4 $\alpha_{58} \beta_{61}$, whereas 3M4E5_T2/anti-CD3 and 3M4E5_T3/anti-CD3 both induced redirected $\mathrm{T}$ cell killing of the $\mathrm{A}^{+}{ }^{+} \mathrm{NY}-\mathrm{ESO}^{-}$antigen-negative cell lines HEP-G2, Ren8, and HISMC at $\mathrm{EC}_{50} \mathrm{~s}$ similar to those for the $\mathrm{A}^{+} \mathrm{NY}-\mathrm{ESO}-1^{+}$cell line NCIH-1755. Similarly, Hyb3.3/anti-CD3 demonstrated $\mathrm{T}$ cell redirected killing of multiple $\mathrm{A1}^{+} \mathrm{MAGE}-\mathrm{A} 1^{-}$ antigen-negative cell lines as well as $\mathrm{A} 1^{+} \mathrm{MAGE}-\mathrm{A} 1^{+}$antigen-positive cell lines, demonstrating no, or a very small, specificity window. In contrast, IMC-MAG-IC3-mediated redirected $\mathrm{T}$ cell killing of $\mathrm{A1}^{+} \mathrm{MAGE}-\mathrm{A3}^{-}$antigen-negative cells was absent (HISMC cells) or only occurred at very high concentrations (COLO205), demonstrating a clear specificity window compared with redirected $\mathrm{T}$ cell killing against $\mathrm{A1}^{+} \mathrm{MAGE}-\mathrm{A3}^{+}$antigen-positive cells (Figure $6 \mathrm{~B}$ and Supplemental Figure 6). These findings were confirmed for both the A2-SLL and the A1-EAD/EVD reactive reagents in 3 additional donors, demonstrating a very similar overall pattern of reactivity 

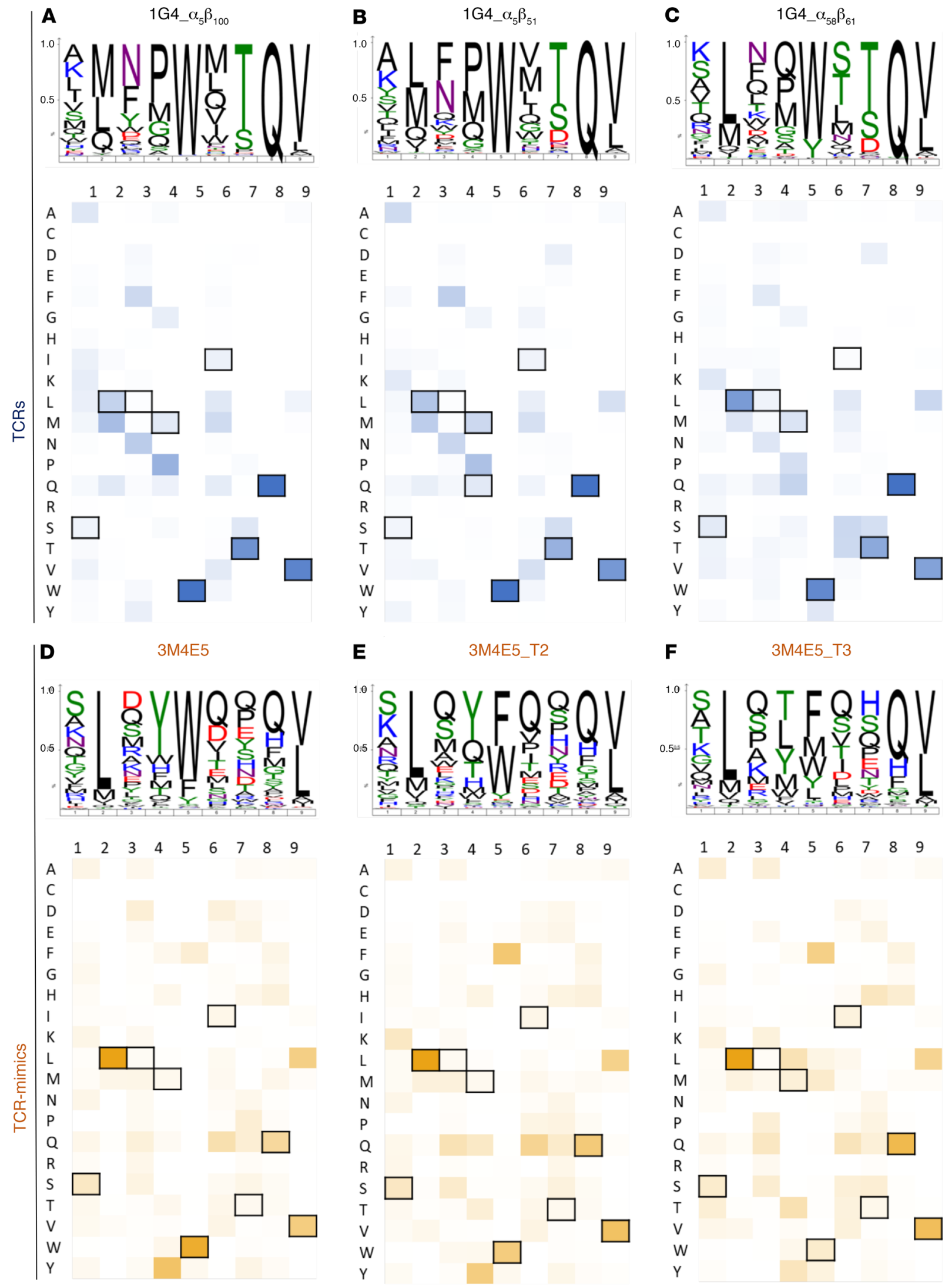

Figure 3. Deep sequencing of peptides from randomized pHLA phage libraries demonstrates the binding degeneracy of pHLA-targeting reagents. Sequence logos (iceLogo software) and heatmaps were generated from NGS sequencing of pan 3 data identifying the distribution of amino acid identities per position of the peptide selected by A2-SLL-reactive affinity-enhanced TCRs and TCR-mimic antibodies. The abundance of an amino acid is shown by intensity of color. Outlined boxes identify the amino acids of the cognate antigen SLL. Data are shown as the average of 2 experimental repeats. (A) $1 \mathrm{C} 4 \_\alpha_{5} \beta_{100}$, (B) $1 \mathrm{C} 44_{2} \alpha_{5} \beta 5_{1}$, (C) $1 \mathrm{C} 4{ }_{-} \alpha_{58} \beta_{61}$, (D) 3M4E5, (E) 3M4E5_T2, and (F) 3M4E5_T3. 
A

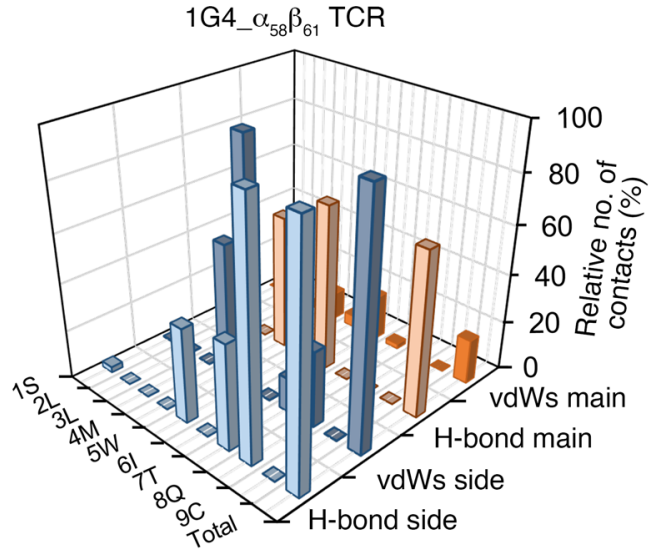

B

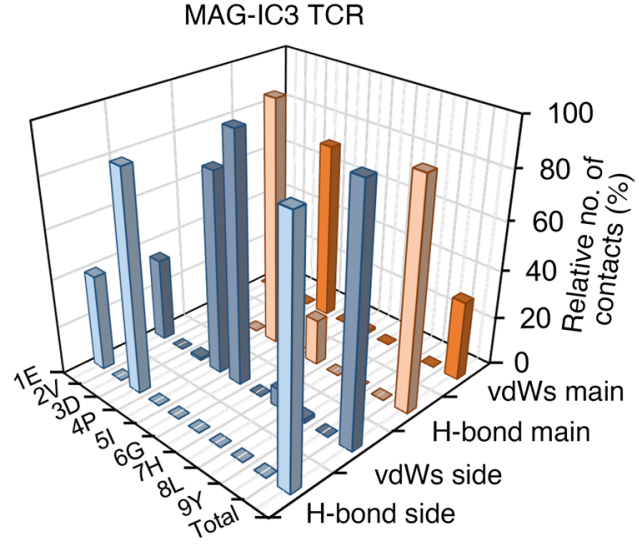

c

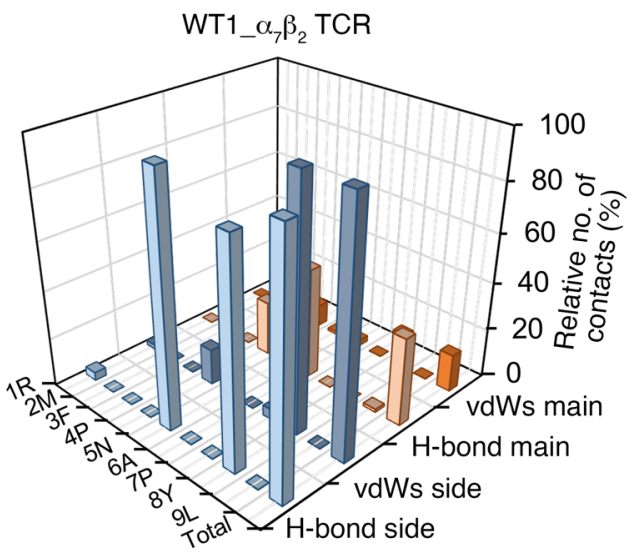

D

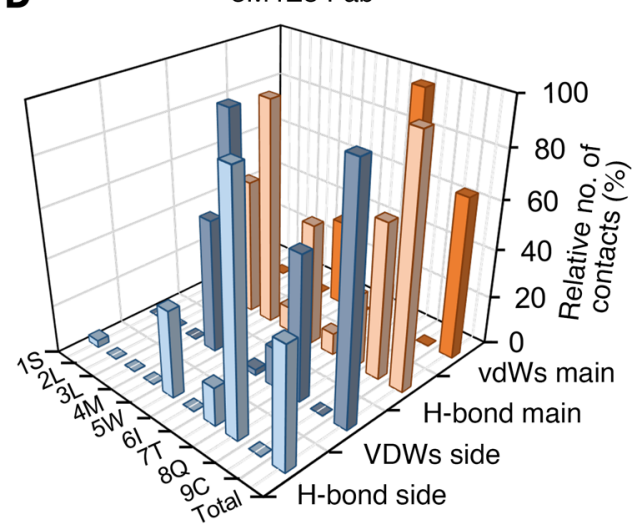

E

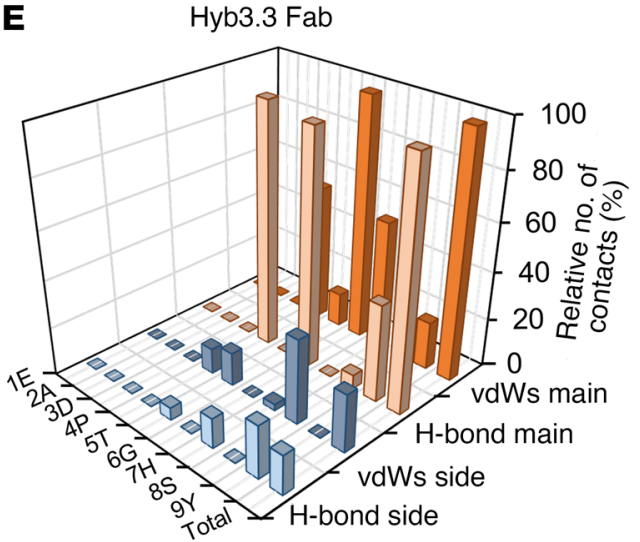

$\mathbf{F}$

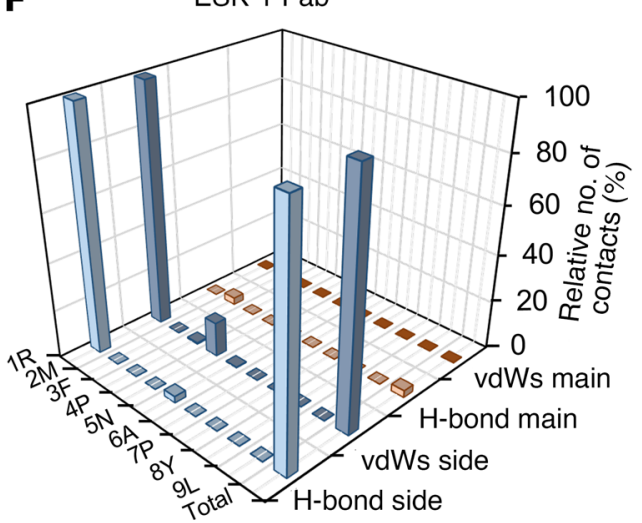

Figure 4. Molecular dynamics simulations reveal broad sidechain contacts with the peptide drive specificity. Relative number of $\mathrm{H}$-bonds and vdW interactions formed between either the main or side chain of each peptide residue to the TCR/TCR mimic over the course of our MD simulations. Total side- versus main-chain ratios for $\mathrm{H}$-bonds and vdW interactions are shown, with the larger value (side or main for each category) scaled to $100 \%$ (absolute values for all contacts are provided in Supplemental Figure 2). MD simulations were performed using independent (random velocity vectors assigned upon heating) 500 ns long MD simulations for each structure. (A) 1C4_ $\alpha_{58} \beta_{61}$-A2SLL, (B) MAG-IC3-A1-EVD, (C) WT1_ $\alpha_{7} \beta_{2}-A 2-R M F,(D)$ 3M4E5A2-SLL, (E) Hyb3.3-A1-EAD, (F) ESK-1-A2-RMF.
(Supplemental Figure 4). Finally, we explored whether ImmTAC molecules might generate a different activation signal in $\mathrm{CD}^{+}$and $\mathrm{CD}^{+} \mathrm{T}$ cells compared with the TCR-mimic antibodies. Purified $\mathrm{CD}^{+}$or $\mathrm{CD}^{+} \mathrm{T}$ cells from a healthy donor were redirected against $\mathrm{A}^{+}{ }^{+} \mathrm{NY}_{-} \mathrm{ESO}^{+}$and $\mathrm{A} 2^{+} \mathrm{NY}^{-E S O}{ }^{-}$target cells using either IMC-1G4 $\alpha_{58} \beta_{61}$ or $3 \mathrm{M} 4 \mathrm{E} 5$ _T3/anti-CD3, and the production of cytokines was measured using a Human TH1/TH2 10-Plex Tissue Culture Kit (Supplemental Figure 7). Overall, the cytokine profiles for both reagents were consistent with an IFN- $\gamma$-driven Th1 CD4 ${ }^{+} \mathrm{T}$ cell and effector $\mathrm{CD}^{+} \mathrm{T}$ cell response, while all other cytokines tested were only detectable at very low levels. We previously reported a similar pattern of activation for $\mathrm{CD}^{+}$and $\mathrm{CD} 4^{+} \mathrm{T}$ cells upon redirection with ImmTAC molecules (59), and together with these findings, this indicates that the mode of $\mathrm{T}$ cell activation is not affected by the selectivity of these bispecifics during $\mathrm{T}$ cell redirection. Furthermore, we observed no cytokine release for IMC-1G4 $\alpha_{58} \beta_{61}$ in response to $\mathrm{A}^{+} \mathrm{NY}^{-\mathrm{ESO}^{-}}$targets, while for 3M4E5_T3/anti-CD3, responses to $\mathrm{A}^{+} \mathrm{NY}^{-} \mathrm{ESO}^{-}$targets were observed for virtually all cytokines tested. This target-independent activation may also explain why 3M4E5_T3/anti-CD3 induced greater cytokine release against the $\mathrm{A} 2{ }^{+} \mathrm{NY}-\mathrm{ESO}^{+}$targets compared with IMC-1G4_ $\alpha_{58} \beta_{61}$, as responses to antigens other than A2-SLL for 3M4E5_T3/anti-CD3 may have resulted in an increase in target abundance for this reagent.

Overall, the HLA-targeting bispecifics based on the natural TCR scaffold retained higher levels of specificity in cellular testing, consistent with the MagPix and randomized pHLA library 
A



B

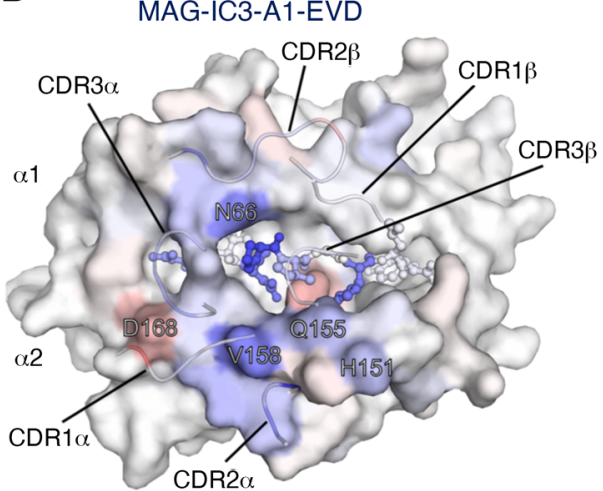

C

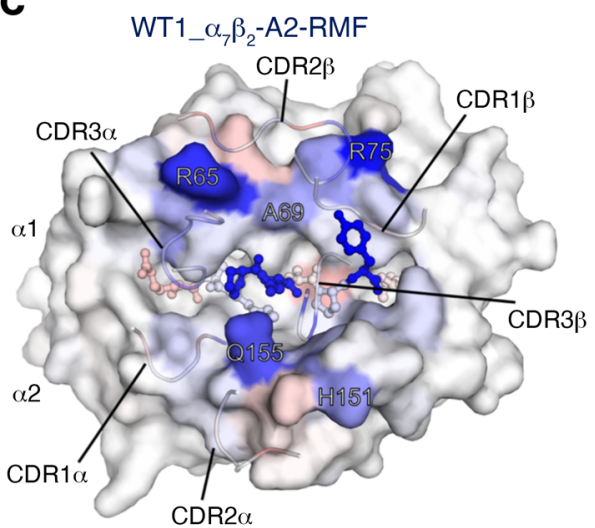

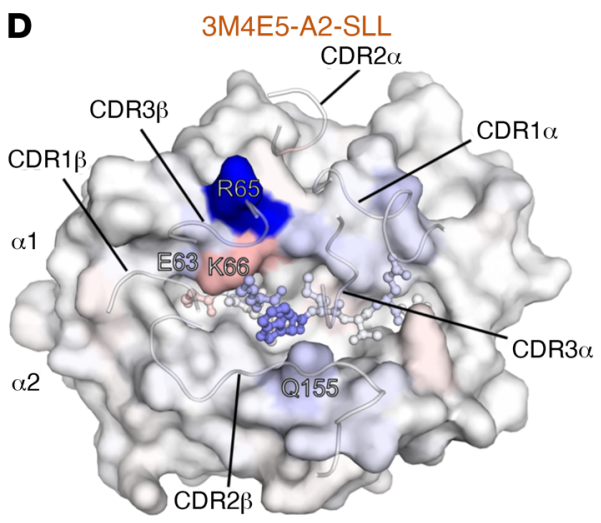

E

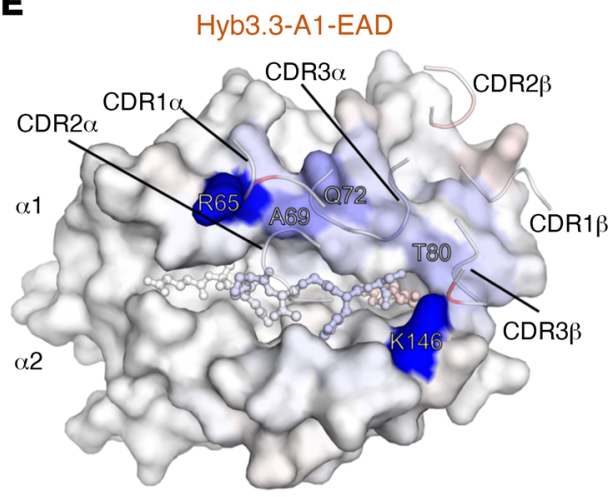

$\mathbf{F}$

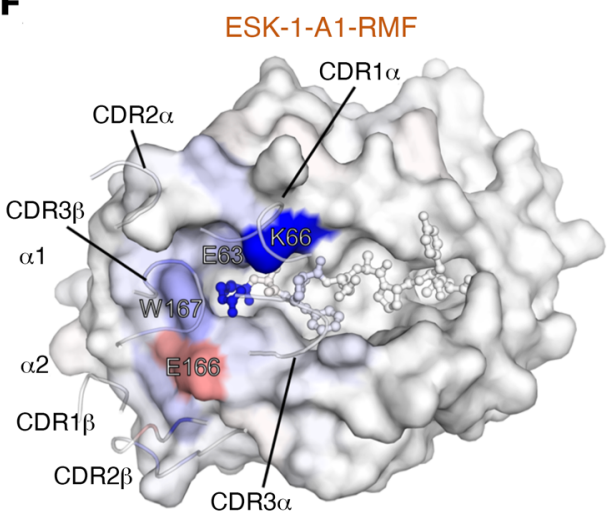

Figure 5. Binding free energy decomposition analysis of TCR and TCR mimic-pHLA interactions. Per residue decomposition of the binding free energy obtained from our MMPBSA calculations to identify energetic hot spots for each TCR or TCR mimic interaction with cognate pHLA. A topdown view of each pHLA is shown, with the peptide depicted as sticks and the HLA as a surface. Color mapping of the decomposition results for each residue was performed across the entire binding interface and used to indicate which residues across this interface favor (blue) or disfavor (red) binding (with white indicating no preference). Bar graphs for all decomposition results are provided in Supplemental Figure 3. MD simulations were performed using 25 independent (random velocity vectors assigned upon heating) 4 ns long MD simulations for each structure. (A) 1G4_ $\alpha_{58} \beta_{61}-\mathrm{A} 2-\mathrm{SLL}$, (B) MAG-IC3-A1-EVD, (C) WT1_ $\alpha_{7} \beta_{2}-A 2-R M F$, (D) 3M4E5-A2-SLL, (E) Hyb3.3-A1-EAD,

(F) ESK-1-A2-RMF. analyses. These findings support the hypothesis that dispersed peptide contacts with the comparatively broad peptide sidechain-focused energetic signature are predictive of the ability to discriminate between different peptides.

The NYBR1 TCR exhibits no cellular off-target reactivity and utilizes a broad, peptide side-chain-centric binding mode. In order to test the notion that peptide selectivity is associated with broad contacts with peptide side chains and a dispersed energetic profile, we extended our analysis to include an affinity-enhanced TCR that was known to be highly selective for its target pHLA. The NYBR1 TCR was affinity matured against a cancer-specific HLA-A ${ }^{*}$ 2:01 restricted peptide (SLSKILDTV; herein referred to as SLS peptide) derived from the NY-BR-1 lineage antigen and used to generate an ImmTAC molecule (IMC-NYBR1). In cellular testing, as assessed by both IFN- $\gamma$ release and target cell killing, IMC-NYBR1 demon- strated exquisite specificity, as evidenced by absence of $\mathrm{T}$ cell redirection against $10 \mathrm{~A} 2^{+} \mathrm{NY}-\mathrm{BR}-1^{-}$antigen-negative cell lines, even at high concentrations (up to $2 \mathrm{nM}$ of IMC-NYBR1 for a $\mathrm{K}_{\mathrm{D}}=47 \mathrm{pM}$ affinity reagent) (Figure 7A). We solved the structure of the NYBR1A2-SLS complex at $2.3 \AA$ A resolution (Table 1), which demonstrated that NYBR1 bound canonically, with a normal crossing angle $\left(63.6^{\circ}\right)$ and a BSA slightly above the reported range $\left(2835 \AA^{2}\right)$, engaging with 7 of the 9 peptide residues (32\% peptide contacts) (Supplemental Figure 8A). This binding mode enabled fine specificity across the peptide, evidenced by sensitivity to alanine substitutions at every peptide position apart from peptide position 1 (Figure 7B). Analysis of contacts and energetics from MD simulations demonstrated a highly peptide side-chain-mediated interaction (Supplemental Figure 8, B-D), with important contributions from 6 of the 9 residues in the SLS peptide. Although there was a slight energetic focus 

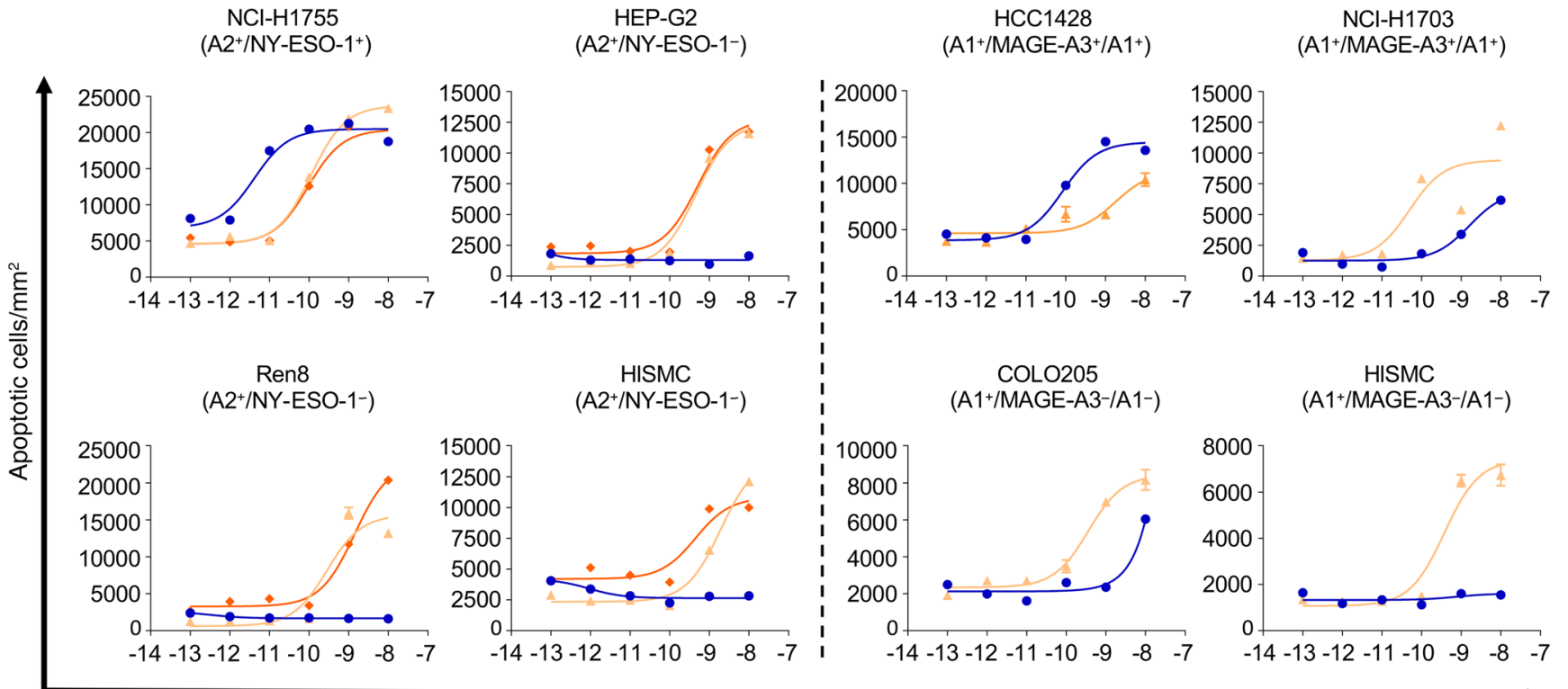

$\log [\mathrm{ImmTAC/scFV}] \mathrm{M}$
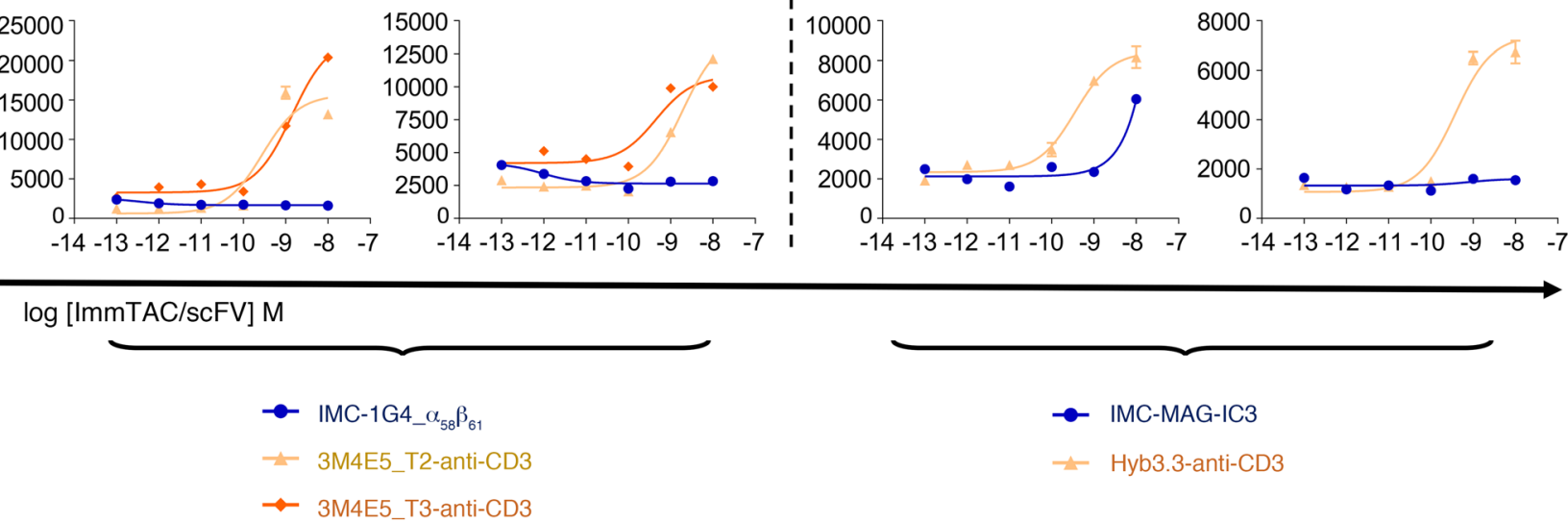

Figure 6. Redirected T cell killing of antigen-positive and -negative cell lines using pHLA-targeting bispecifics. The activity of the ImmTAC molecules and the TCR mimic/anti-CD3 fusions was tested against a range of antigen-positive and antigen-negative cell lines (tumor and healthy cells) using IncuCyte killing assays. PBMCs from 1 healthy donor are shown. PBMCs from an additional 3 healthy donors were used to repeat the assay (Supplemental Figure 4). Data are plotted using area under the curve analysis. Error bars show SD from 3 experimental repeats. (A) IMC-1C4_ $\alpha_{58} \beta_{61}, 3 M 4 E 5$ _T2/anti-CD3, and 3M4E5_T3/anti-CD3 T cell redirection against HLA-A*02:01+NY-ESO-1+ (NCI-H1755) and HLA-A*02:01+NY-ESO-1- (HEP-C2, Ren8, and HISMC) cells lines. (B) IMC-MAG-IC3 and Hyb3.3/anti-CD3 T cell redirection against HLA-A*01:01+MAGE-A3+ (HCC1428 and NCI-H1703), HLA-A*01:01+MACE-A1+ (HCC1428 and NCI-H1703), and HLA-A*01:01+MAGE- (COLO205 and HISMC) cells lines.

toward HLA residue Q155 (-6 kcal mol${ }^{-1}$ ) and peptide residue K4 (-9 $\left.\mathrm{kcal} \mathrm{mol}^{-1}\right)$, a number of energetic contributions were made across the entire HLA surface and peptide. Thus, consistent with our other observations in this study, the fine specificity of the NYBR1 TCR was associated with a broad energetic binding mode characterized by interactions with multiple peptide side chains.

\section{Discussion}

The identification of cancer-specific targets for solid tumors is challenging because cell-surface antigens are often expressed on a wide range of tissues. Molecules such as CD19 and others have proved effective targets for liquid tumors because on-target toxicity is limited to mainly hematopoietic cells that can repopulate from the bone marrow following treatment (60). Another key source of antigens is dysregulated or mutated intracellular proteins. However, many of these proteins are only presented on the cell surface in the context of pHLA. It is interesting to note that, even in response to very common human pathogens (i.e., influenza), natural antibodies recognizing pHLA have not been detected, suggesting that humoral responses to pHLA are either ineffective or dangerous to the host. Unlike antibodies, TCRs are selected to recognize pHLA in the thymus, which deletes T cells with TCRs that bind strongly to self-pHLA (and presumably removes TCRs that bind in a peptide-independent manner). This process is controlled in part by
HLA coengagement by the T cell coreceptors CD8 and CD4, removal of which in CD8/CD4 coreceptor knockout mice has been shown to enable the selection of $\mathrm{T}$ cells with TCRs that can bind to non-MHC proteins, such as CD155 $(61,62)$. A number of recent reports have also suggested that, probably as a consequence of thymic selection, TCRs have coevolved to recognize pHLA (63-65). This "coevolution" model is consistent with findings from a recent study revealing that TCRs have a unique variable domain orientation compared with antibodies, the absence of which might restrict antibodies from reproducing the natural ability of TCRs to discriminate between different HLA-presented peptides (66). Thus, TCRs have multiple unique selection mechanisms and structural features that guide pHLA recognition. We have previously demonstrated that affinity-enhanced TCRs maintain many of the binding characteristics of their thymically selected WT progenitors, with stronger binding generally being driven through the formation of new interactions with both the peptide and HLA surface $(13,44,45$, 67-69). This feature likely provides an advantage for generating affinity-enhanced TCRs with the ability to retain a similar specificity profile compared with natural, thymically selected TCRs.

The literature indicates that $\mathrm{T}$ cell potency is tuned by a TCR affinity threshold that is optimal in the low micromolar to high nanomolar range $(17,34,70-74)$. For soluble bispecific reagents, this affinity threshold is controlled at the effector end of the mol- 
A
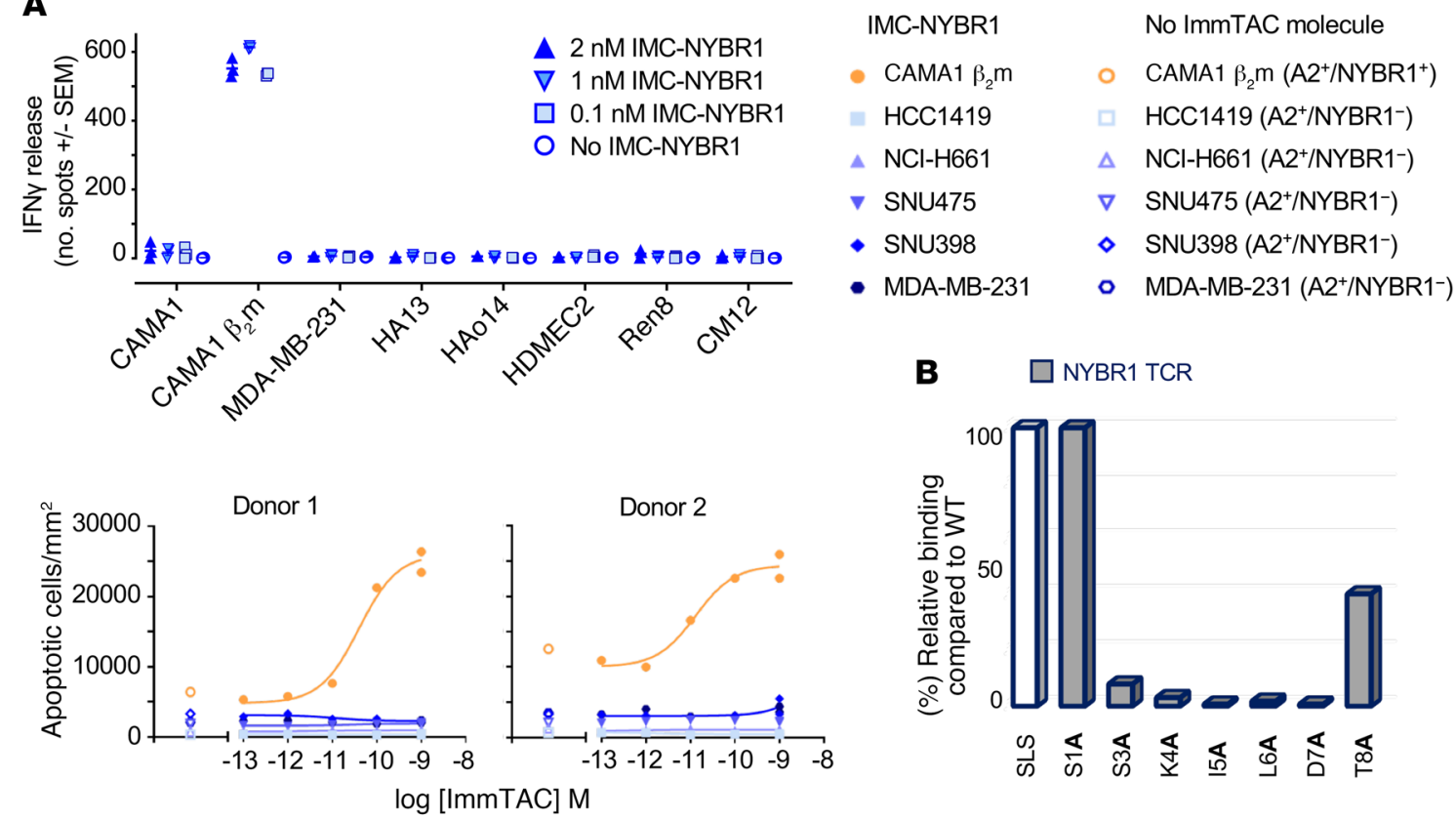

Figure 7. The NYBR1 TCR exhibits no cellular off-target reactivity and is sensitive to alanine substitutions across the peptide backbone. (A) The activity of IMC-NYBR1 was tested against a range of HLA-A*02:01+NYBR1 (CAMA1 and CAMA1 $\beta_{2} m$ ) and HLA-A*02:01+NYBR1- cell lines (MDA-MB-231, HA13, HA014, HDMEC2, Ren8, CM12 HCC1419, NCI-H661, SNU475, and SNU398) using IFN- $\gamma$ ELISpot (bar graphs) and IncuCyte killing assays (area under the curve analysis) in 2 donors. Error bars show SD from 3 experimental repeats. (B) The contribution of peptide side chains to binding specificity was analyzed using alanine scan mutagenesis (by surface plasmon resonance). Binding affinities of the NYBR1-A2-SLS interaction were determined using single-cycle kinetic analysis. Bar graphs show binding affinity as a percentage relative to the binding affinity to the index peptide.

ecule, while the affinity of the pHLA targeting end must be optimized to achieve potency according to receptor occupancy. For most tumor-associated pHLAs, their natural presentation levels can be very low (often below 10 copies of each specific peptide epitope per cell) $(3,33)$. Consequently, binding in the femto- to picomolar affinity range is needed to achieve a therapeutically relevant receptor occupancy level: a feat that has been achieved for monovalent TCR-based bispecifics $(22,24)$. The literature, together with the data presented here, suggests that, with current technologies, engineering a TCR-mimic antibody to achieve this affinity range while maintaining peptide selectivity is more challenging (32). Some soluble TCR mimics have been designed as full antibodies, thus achieving much stronger binding through avidity effects (38). However, this essentially halves the number of effector molecules per target cell, a major issue if antigen presentation levels are already limiting.

Here, we interrogated the molecular basis of pHLA recognition of a panel of $\mathrm{T}$ cell-redirecting bispecifics using a combination of structural, biochemical, and computational approaches. All the affinity-enhanced TCRs utilized a canonical native TCRlike binding mode, maintaining broad contacts across the peptide backbone. This finding likely represents the advantage of using reagents that have been developed from a thymically selected TCR progenitor. Our data also reveal that a native-like binding mode is necessary, but not sufficient, for enabling peptide selectivity. For instance, despite the 3M4E5 TCR mimic binding in an almost identical fashion to a native TCR, with peptide contacts across the peptide backbone, it still performed poorly in cellular crossreactivity assays. Our MD simulation analysis demonstrated that 3M4E5 bound via 2 main hot spots: 1 on peptide residue W5 and 1 on HLA residue R65. These findings were mirrored in the randomized pHLA library analysis that demonstrated the A2-SLL-reactive TCR mimics were all more degenerate (in terms of amino acid preferences across the peptide backbone) as compared with the A2-SLL-reactive affinity-enhanced TCRs and were predicted to select a far larger number of unique peptide sequences. Interestingly, this hot spot-mediated binding mode has also been observed for some natural TCRs, but has been shown to correlate with high levels of TCR crossreactivity and has been implicated in autoimmunity $(49,75)$. The ESK- 1 and Hyb3.3 TCR mimics also employed hot spot-binding modes, especially focused toward residues on the HLA surface, and demonstrated loss of peptide selectivity in biochemical (ESK-1 and Hyb3.3) and cellular (Hyb3.3) testing. In contrast, the TCR-based reagents tested all exhibited superior peptide selectivity in biochemical and cellular tests and were characterized by binding modes that included a greater combination of balanced energetic interactions across the peptide and HLA surfaces. This was exemplified by the NYBR1 TCR (by far the cleanest molecule tested in both the molecular and cellular analysis), which also demonstrated a broad energetic binding mode, with the majority of peptide contacts through side-chain interactions. Although the TCR mimics selected in this study were generated using a different approach from the affinityenhanced TCRs, our data suggest that the reagents based on the natural TCR scaffold were better able to discriminate between different peptides by utilizing peptide-specific binding interactions.

Even though we focused on 3 published TCR-mimic antibodies because of available structures $(25,31,36)$, our data also have impli- 
cations for other published studies of TCR mimics. For instance, the TCR mimic ESK-1 has been shown to target the Wilms tumor antigen in mouse models $(29,38)$, despite evidence demonstrating that the A2-RMF antigen is not expressed (58). This finding, combined with the data shown here, suggests that the activities reported for ESK-1 may be mediated by recognition of another peptide or in a peptide-independent fashion. Although it is clear that engineering pHLA selectivity is still one of the major challenges for TCR mimics (76), there are emerging reagents that exhibit more promising specificity profiles, including reagents targeting an $\mathrm{HLA}^{*} \mathrm{~A}^{*}$ 2:01 restricted EBV LMP2A ${ }_{426-434}$ (CLGGLLTMV) epitope (77) and an $\alpha$-fetoprotein ${ }_{158-166}$ (FMNKFIYEI) epitope (78), the second of which has entered clinical trials as a CAR for the treatment of liver cancer. Our data demonstrate the importance of robust specificity testing of pHLA-targeting molecules, in line with our own preclinical safety testing package (79), that should be considered for the development of soluble pHLA-targeting bispecifics.

In summary, we demonstrate that by combining structural and biochemical data with atomistic MD simulations, the interactions underpinning pHLA recognition can be dissected in detail and can be used to better understand the specificity of pHLAtargeting reagents. Although these findings need to be further validated using in vivo tumor models, they extend our understanding of the molecular rules that determine selective recognition of pHLA and shed additional light on how TCRs engage pHLA in a peptide-dependent fashion. Finally, these observations also highlight the challenges associated with engineering pHLA-targeting molecules that can truly mimic TCR-like specificity.

\section{Methods}

Cell lines. COLO205, HCC1428, NCI-H1703, NCI-H1755, NCI-H1944, NCI-H2087, NCI-H441, and T2 antigen-presenting cells (LCL721 × CEM-C7) were purchased from ATCC and cultured in RPMI supplemented with 10\% FCS, 2 mM L-glutamine, and 1\% (v/v) penicillin/streptomycin. HEP-G2 cells were purchased from ATCC and cultured in EMEM supplemented with 10\% FCS. HISMC and HREpic8 (Ren8) cells were purchased from ScienCell and cultured in smooth muscle cell medium or epithelial cell medium, respectively. Cell line authentication and mycoplasma testing were routinely carried out by the LGC Standards Cell Line Authentication Service (www.lgcstandards.com) and Mycoplasma Experience Ltd. (www.mycoplasma-exp.com), respectively.

TCR engineering. To obtain TCRs with affinity enhanced for HLA-A ${ }^{*}$ 2:01 SLLMWITQC (NY-ESO- $1_{157-165}$ ), HLA-A ${ }^{*} 01: 01$ EVDPIGHLY (MAGE-A3 ${ }_{161-134}$ ), and HLA-A ${ }^{*}$ 2:01 RMFPNAPYL (WT- $1_{126-134}$ ), the WT 1G4, MAGE-A3, and WT1 TCRs were subjected to phage display, as previously described (24). A panel of high-affinity TCRs were obtained with mutations in the $\alpha$ and/or the $\beta$ chain (data not shown). TCRs from these panels were selected for this study according to their similarity in affinity to available TCR-mimic antibodies. Additionally, some stronger affinity-engineered TCRs were selected according to the availability of corresponding TCR-pHLA complex structures to enable direct structural comparisons with the TCR-mimic antibodies.

Construct design, protein expression, and purification. The modified TCRs, the TCR mimics, $\beta_{2} \mathrm{~m}$, and the HLA- $\mathrm{A}^{*} 01: 01$, and HLA-A ${ }^{*}$ 2:01 heavy chains were cloned into the pGMT7 vector and expressed in the BL21 (DE3) Rosetta pLysS E.coli strain as described previously $(16,80)$.
TCR constructs for biophysical analysis were designed to include the variable and constant domains of both chains ( $\alpha$ and $\beta$ ) with an engineered interchain disulphide bond (23). Antibody reagents for biophysical analysis were generated as $\mathrm{scFv}$ with a linker between the variable heavy and light chains (the constant domains were not included in the construct). Hyb3.3 scFv expression cassette was cloned into pCEP4 and protein expressed in mammalian cells using the ExpiCHO Expression System (Thermo Fisher Scientific), as it did not express in E. coli. TCR constructs for T cell redirection experiments were generated with an anti-CD3 scFv fused to the TCR- $\beta$ chain (ImmTAC molecules, total size $\sim 75 \mathrm{kDa}$ ) (22). TCR-mimic antibody constructs for T cell redirection experiments were designed as $\mathrm{scFv}$ with an anti-CD3 scFv fused to the heavy chain (total size $\sim 50 \mathrm{kDa}$ ).

Analysis of on-target and off-target $T$ cell reactivity via redirection using anti-pHLA/anti-CD3 bispecific reagents. The activity of the ImmTAC molecules (TCR-CD3 scFv fusions) and the TCR mimic $\mathrm{scFv}-\mathrm{CD} 3 \mathrm{scFv}$ fusions was tested through their ability to redirect $\mathrm{T}$ cells against a range of antigen-positive and antigen-negative cell lines (tumor and healthy cells). IncuCyte killing assays were performed according to the manufacturer's instructions (Sartorius). For multiplex cytokine analysis, healthy donor $\mathrm{CD}^{+}$and $\mathrm{CD}^{+} \mathrm{T}$ cells were isolated by magnetic separation using negative selection kits following the manufacturer's instructions (Miltenyi).

MagPix peptide screening. Affinity-enhanced TCRs and TCR mimic scFvs were subjected to peptide crossreactivity analysis using a MagPlex bead kit (Invitrogen). Several common peptides expressed by healthy cells were refolded with either biotin-tagged HLA-A ${ }^{\star} 02: 01$ or biotin-tagged HLA-A ${ }^{*} 01: 01$ (detailed in Tables 3 and 4). Phagemid-encoded TCRs/TCR mimics were expressed on the surface of bacteriophage M13, fused to capsid protein pIII (81), and binding to biotinylated self-peptide-HLA complexes attached to neutravidin-conjugated MagPlex magnetic beads assayed. Positively bound beads were identified by MagPix analysis using a phagespecific PE-conjugated anti-M13 bacteriophage coat protein g8p antibody (RL-ph2, 2B Scientific Limited, MUB0604) conjugated to R-phycoerythrin using a Conjugation Kit (Abcam, ab102918). Peptides generating a signal above background (3 times median intensity of all bead regions bound to native helper phage) were classified as positive binders and binding expressed as a percentage of signal obtained relative to index peptide. Averages of triplicate measurements for each interaction were determined within each experiment, and percentage binding for each interaction is reported as the average of several experimental repeats.

SPR single-cycle kinetic analysis. Purified TCRs and TCR mimic scFvs were subjected to SPR analysis using a BIAcore 3000 with singlecycle kinetic analysis, as previously reported $(67,69)$.

Panning using scHLA libraries. scHLA libraries were generated as previously described (50). Biotinylated affinity-enhanced TCRs $\left(1 G 4_{-} \alpha_{5} \beta_{100}, 1 G 4 \alpha_{-} \alpha_{5} \beta_{51}\right.$, and $\left.1 G 4 \alpha_{-} \alpha_{58} \beta_{61}\right)$ and TCR-mimic antibodies (3M4E5, 3M4E5_T2, and 3M4E5_T3) were captured on streptavidincoated paramagnetic beads and incubated with the library of purified phage particles preblocked in 3\% MPBS buffer. Phage particles were eluted in trypsin and used to infect early log phase TGI E. coli cells and plated onto YTEag plates at $30^{\circ} \mathrm{C}$ for 16 hours. Three rounds of selection were performed.

Deep sequencing of pHLA libraries. DNA was isolated from each glycerol stock by Miniprep (Zymoprep II Kit, Zymo Research). 
Sequencing libraries were prepared with molecular indexing based on a method described previously (82). Libraries were sequenced using Illumina V3 SBS chemistry on the MiSeq sequencer. Basecalling and sample demultiplexing were performed using MiSeq reporter to generate fastq files and were processed with custom analysis pipeline. Peptide repertoire analysis was performed using Excel, and sequence logos were generated using iceLogo standalone tool (83). Sequence clustering analysis was performed with GibbsCluster, version 2.0, web server using default settings (84).

$X$-ray crystallography. Crystals were grown at $18^{\circ} \mathrm{C}$ by vapor diffusion via the sitting drop technique. All crystallization screening and optimization experiments were completed with an Art Robbins Phoenix Dispensing Robot (Alpha Biotech Ltd.). $200 \mathrm{~nL}$ of 10 to $15 \mathrm{mg} / \mathrm{mL}$ TCR-pHLA complex mixed at a 1:1 molar ratio was added to $200 \mathrm{~nL}$ of reservoir solution. INTELLI-PLATES were then sealed and incubated in a crystallization incubator at $18^{\circ} \mathrm{C}$ (RuMed, Rubarth Apperate $\mathrm{GmbH}$ ) and analyzed for crystal formation using a Rock Imager 2 (Formulatrix). Crystals selected for further analysis were cryoprotected with ethylene glycol to $25 \%$ and then flash-cooled in liquid nitrogen in Litho loops (Molecular Dimensions). For WT1_ $\alpha_{7} \beta_{2}-\mathrm{A} 2-\mathrm{RMF}$, optimal crystals were obtained in 0.1 M HEPES pH 7, 0.1M ammonium, 20\% v/v Sok-CP7. For NYBR1-A2-SLS, optimal crystals were obtained in Pact premier (Molecular Dimensions) condition B07 (0.2 M sodium chloride, pH 6.0, 0.1 M MES, and 20\% PEG 6000). Diffraction data were collected at several different beamlines at the Diamond Light Source using a Dectris Pilatus 6M detector. Using the rotation method, 1000 frames were recorded, each covering $0.2^{\circ}$ of rotation. Reflection intensities were estimated with the XIA2 package $(45,46)$, and the data were scaled, reduced, and analyzed with AIMLESS and the CCP4 package (47). TCR/pHLA complex structures were solved with molecular replacement using Phaser (49), using PDB 4I4W as a starting model for pHLA and PDB 3O4L as a starting model for NYBR1 TCR. Accession code for the crystal structures reported in this study have been uploaded to the RCSB's Protein Data Bank database (PDB WT1_ $\alpha_{7} \beta_{2}$-A2-RMF: 6RSY and NYBR1-A2-SLS: 6R2L).

$M D$ simulations and MMPBSA calculations. Periodic boundary simulations and MMPBSA calculations were performed with the Amber16 suite of programs (85). X-ray crystal structures of the 7 TCR/Fab-pHLA complexes investigated were used as the starting points for MD simulations. Missing residues were added using Modeller, version 9 (86). MolProbity was used to modify histidine tautomerization states (tautomerization states used can be found in Supplemental Table 2) and Asn/Gln side-chain orientations under the criteria of optimizing the internal H-bond network. PropKa, version 3.0 (87), was used to check the likely protonation states of all titratable residues for $\mathrm{pH} 7$ (all residues were modeled in their standard protonation states). Each system was solvated in a rectangular box of water (with all crystallographic waters retained) extending at least $10 \AA$ away from any protein atom. Sodium or chloride ions were added as necessary to neutralize the total system charge. The ff14SB (88) and TIP3P (89) force fields were used to describe protein and water molecules, respectively. Following minimization, heating, and equilibration (see Supplemental Methods section Structure equilibration procedure), each system was subjected to two 500 ns long production MD simulations (random velocity vectors assigned upon heating) in the NPT ensemble ( $1 \mathrm{~atm}, 298 \mathrm{~K})$. Production MD simulations were run with the SHAKE algorithm applied, a $2 \mathrm{fs}$ time step, and a collision frequency of $1 \mathrm{ps}^{-1}$. An $8 \AA$ direct space nonbonded cutoff was applied with long-range electrostatics evaluated using the particle mesh Ewald algorithm (90). H-bond (including water bridged hydrogen bonds) and vdW interactions were evaluated from snapshots saved every $10 \mathrm{ps}$, using the last $450 \mathrm{~ns}$ of each trajectory (900 ns per TCR/Fab-pHLA). An H-bond was defined as on if the donor acceptor distance was within $3.0 \AA$ and the donor hydrogen acceptor angle was within $45^{\circ}$ to $180^{\circ}$. A vdW interaction was defined as on if 2 heavy atoms were within $4 \AA$ of one another.

Statistics. Detailed methodology is described in the Structure equilibration procedure section of Supplemental Methods.

Study approval. PBMCs were obtained from healthy volunteers. The Oxford A REC-approved protocol 13/SC/0226 was used to obtain written consent for all blood donations and was fully approved by the National Research Ethics Committee South Central.

\section{Author contributions}

CJH, RMC, JMP, BDW, AL, N Lissin, EAP, KAL, THB, SH, PJC, MH, RJP, TES, BC, AJ, PEM, AV, MS, MA, N Liddy, RAR, SH, VS, ML, CRP, MWVDK, PJR, BKJ, and DKC performed and/or directed experiments, analyzed data, and critiqued the manuscript. DKC and BKJ conceived and directed the project. DKC wrote the manuscript.

\section{Acknowledgments}

The authors would like to thank Diamond Light Source for beam time and the staff for assistance with crystal testing and data collection. The authors would also like to thank Kate Lowe, Ita O'Kelly, and Michelle McCully for critically reading the manuscript. RMC's studentship is funded by the Engineering and Physical Sciences Research Council (EPSRC). MWVDK is a Biotechnology and Biological Sciences Research Council (BBSRC) David Phillips Fellow (BB/M026280/1). This research made use of the Balena High Performance Computing (HPC) Service at the University of Bath as well as the computational facilities of the Advanced Computing Research Centre of the University of Bristol.

Address correspondence to: David Cole and Annelise Vuidepot, 91 Park Drive, Milton Park, Abingdon, Oxfordshire, OX14 4RY, United Kingdom. Phone: 44.1235.776256, 44.1235.438668; Email: david. cole@immunocore.com, annelise.vuidepot@immunocore.com.
1. Comber JD, Philip R. MHC class I antigen presentation and implications for developing a new generation of therapeutic vaccines. Ther $A d v$ Vaccines. 2014;2(3):77-89.

2. Heemskerk B, Kvistborg P, Schumacher TN. The cancer antigenome. EMBO J. 2013;32(2):194-203.
3. Garrido F, Cabrera T, Aptsiauri N. "Hard" and "soft" lesions underlying the HLA class I alterations in cancer cells: implications for immunotherapy. Int J Cancer. 2010;127(2):249-256.

4. Berah M, Hors J, Dausset J. A study of HL-A antigens in human organs. Transplantation.
1970;9(3):185-192.

5. Williams KA, Hart DN, Fabre JW, Morris PJ. Distribution and quantitation of HLA-ABC and DR (Ia) antigens on human kidney and other tissues. Transplantation. 1980;29(4):274-279.

6. Brameshuber M, et al. Monomeric TCRs drive 
T cell antigen recognition. Nat Immunol. 2018;19(5):487-496.

7. Irvine DJ, Purbhoo MA, Krogsgaard M, Davis MM. Direct observation of ligand recognition by T cells. Nature. 2002;419(6909):845-849.

8. Purbhoo MA, Irvine DJ, Huppa JB, Davis MM. $\mathrm{T}$ cell killing does not require the formation of a stable mature immunological synapse. Nat Immunol. 2004;5(5):524-530.

9. Rossjohn J, Gras S, Miles JJ, Turner SJ, Godfrey DI, McCluskey J. T cell antigen receptor recognition of antigen-presenting molecules. Annи Rev Immunol. 2015;33:169-200.

10. Mason D. A very high level of crossreactivity is an essential feature of the T-cell receptor. Immunol Today. 1998;19(9):395-404.

11. Sewell AK. Why must T cells be cross-reactive? Nat Rev Immunol. 2012;12(9):669-677.

12. Birnbaum ME, et al. Deconstructing the peptide-MHC specificity of T cell recognition. Cell. 2014;157(5):1073-1087.

13. Cole DK, et al. Structural mechanism underpinning cross-reactivity of a CD8+ T-cell clone that recognizes a peptide derived from human telomerase reverse transcriptase. J Biol Chem. 2017;292(3):802-813.

14. Wooldridge $\mathrm{L}$, et al. A single autoimmune $\mathrm{T}$ cell receptor recognizes more than a million different peptides. J Biol Chem. 2012;287(2):1168-1177.

15. Aleksic M, et al. Different affinity windows for virus and cancer-specific T-cell receptors: implications for therapeutic strategies. Eur J Immunol. 2012;42(12):3174-3179.

16. Cole DK, et al. Human TCR-binding affinity is governed by MHC class restriction. J Immunol. 2007;178(9):5727-5734.

17. Schmid DA, et al. Evidence for a TCR affinity threshold delimiting maximal CD8 $\mathrm{T}$ cell function. JImmunol. 2010;184(9):4936-4946.

18. Rapoport AP, et al. NY-ESO-1-specific TCRengineered $T$ cells mediate sustained antigen-specific antitumor effects in myeloma. Nat Med. 2015;21(8):914-921.

19. Rohaan MW, Wilgenhof S, Haanen JBAG. Adoptive cellular therapies: the current landscape. Virchows Arch. 2019;474(4):449-461.

20. Baruch EN, Berg AL, Besser MJ, Schachter J, Markel G. Adoptive T cell therapy: An overview of obstacles and opportunities. Cancer. 2017;123(S11):2154-2162.

21. Husain B, Ellerman D. Expanding the boundaries of biotherapeutics with bispecific antibodies. BioDrugs. 2018;32(5):441-464.

22. Liddy N, et al. Monoclonal TCR-redirected tumor cell killing. Nat Med. 2012;18(6):980-987.

23. Boulter JM, et al. Stable, soluble T-cell receptor molecules for crystallization and therapeutics. Protein Eng. 2003;16(9):707-711.

24. Li Y, et al. Directed evolution of human T-cell receptors with picomolar affinities by phage display. Nat Biotechnol. 2005;23(3):349-354.

25. Ataie N, et al. Structure of a TCR-mimic antibody with target predicts pharmacogenetics. J Mol Biol. 2016;428(1):194-205.

26. Biddison WE, Turner RV, Gagnon SJ, Lev A, Cohen CJ, Reiter Y. Tax and M1 peptide/ HLA-A2-specific Fabs and T cell receptors recognize nonidentical structural features on peptide/HLA-A2 complexes. JImmunol. 2003;171(6):3064-3074.

27. Chames $P$, et al. TCR-like human antibodies expressed on human CTLs mediate antibody affinity-dependent cytolytic activity.J Immunol. 2002;169(2):1110-1118.

28. Chang AY, et al. A therapeutic $\mathrm{T}$ cell receptor mimic antibody targets tumor-associated PRAME peptide/HLA-I antigens. JClin Invest. 2017;127(7):2705-2718.

29. Dao T, et al. Therapeutic bispecific T-cell engager antibody targeting the intracellular oncoprotein WT1. Nat Biotechnol. 2015;33(10):1079-1086.

30. Held G, et al. MHC-peptide-specific antibodies reveal inefficient presentation of an HLA-A ${ }^{*}$ 201-restricted, Melan-A-derived peptide after active intracellular processing. Eur $J$ Immunol. 2007;37(7):2008-2017.

31. Hülsmeyer M, et al. A major histocompatibility complex-peptide-restricted antibody and $t$ cell receptor molecules recognize their target by distinct binding modes: crystal structure of human leukocyte antigen (HLA)-A1-MAGE-A1 in complex with FAB-HYB3. J Biol Chem. 2005;280(4):2972-2980.

32. Maus MV, et al. An MHC-restricted antibody-based chimeric antigen receptor requires TCR-like affinity to maintain antigen specificity. Mol Ther Oncolytics. 2016;3:1-9.

33. Michaeli $\mathrm{Y}$, et al. Expression hierarchy of $\mathrm{T}$ cell epitopes from melanoma differentiation antigens: unexpected high level presentation of tyrosinase-HLA-A2 Complexes revealed by peptide-specific, MHC-restricted, TCR-like antibodies. J Immunol. 2009;182(10):6328-6341.

34. Oren R, et al. Functional comparison of engineered $T$ cells carrying a native TCR versus TCRlike antibody-based chimeric antigen receptors indicates affinity/avidity thresholds. JImmunol. 2014;193(11):5733-5743.

35. Sastry KS, et al. Targeting hepatitis B virusinfected cells with a T-cell receptor-like antibody. J Virol. 2011;85(5):1935-1942.

36. Stewart-Jones G, et al. Rational development of high-affinity T-cell receptor-like antibodies. Proc Natl Acad Sci U S A. 2009;106(14):5784-5788.

37. Yamano $Y$, et al. Increased expression of human $T$ lymphocyte virus type I (HTLV-I) Tax11-19 peptide-human histocompatibility leukocyte antigen $A^{*} 201$ complexes on CD 4+ CD25+ T Cells detected by peptide-specific, major histocompatibility complex-restricted antibodies in patients with HTLV-I-associated neurologic disease. J Exp Med. 2004;199(10):1367-1377.

38. Zhao Q, et al. Affinity maturation of T-cell receptor-like antibodies for Wilms tumor 1 peptide greatly enhances therapeutic potential. Leukemia. 2015;29(11):2238-2247.

39. Abelin JG, et al. Mass spectrometry profiling of HLA-associated peptidomes in mono-allelic cells enables more accurate epitope prediction. Immunity. 2017;46(2):315-326.

40. Hassan C, et al. The human leukocyte antigen-presented ligandome of $\mathrm{B}$ lymphocytes. Mol Cell Proteomics. 2013;12(7):1829-1843.

41. Ritz D, Gloger A, Weide B, Garbe C, Neri D, Fugmann T. High-sensitivity HLA class I peptidome analysis enables a precise definition of peptide motifs and the identification of peptides from cell lines and patients' sera. Proteomics. 2016;16(10):1570-1580.

42. Shao W, et al. The SysteMHC Atlas project. Nucleic Acids Res. 2018;46(D1):D1237-D1247.

43. Dhanik A, et al. In-silico discovery of cancerspecific peptide-HLA complexes for targeted therapy. BMC Bioinformatics. 2016;17:286.

44. Dunn SM, et al. Directed evolution of human T cell receptor $\mathrm{CDR} 2$ residues by phage display dramatically enhances affinity for cognate peptide-MHC without increasing apparent crossreactivity. Protein Sci. 2006;15(4):710-721.

45. Sami M, et al. Crystal structures of high affinity human T-cell receptors bound to peptide major histocompatibility complex reveal native diagonal binding geometry. Protein Eng Des Sel. 2007;20(8):397-403.

46. Kumar P, Vahedi-Faridi A, Saenger W, Ziegler A, Uchanska-Ziegler B. Conformational changes within the HLA-A1:MAGE-A1 complex induced by binding of a recombinant antibody fragment with TCR-like specificity. Protein Sci. 2009;18(1):37-49.

47. Raman MC, et al. Direct molecular mimicry enables off-target cardiovascular toxicity by an enhanced affinity TCR designed for cancer immunotherapy. Sci Rep. 2016;6:18851.

48. Adams JJ, et al. Structural interplay between germline interactions and adaptive recognition determines the bandwidth of TCR-peptide-MHC cross-reactivity. Nat Immunol. 2016;17(1):87-94.

49. Cole DK, et al. Hot spot autoimmune T cell receptor binding underlies pathogen and insulin peptide cross-reactivity. JClin Invest. 2016;126(6):2191-2204.

50. Coles $\mathrm{CH}$, et al. TCRs with distinct specificity profiles use different binding modes to engage an identical peptide-HLA complex [published online February 26, 2020]. JImmunol. https:// doi.org/10.4049/jimmunol.1900915.

51. Miller BR, McGee TD, Swails JM, Homeyer N, Gohlke H, Roitberg AE. MMPBSA.py: An efficient program for end-state free energy calculations. JChem Theory Comput. 2012;8(9):3314-3321.

52. Genheden S, Ryde U. The MM/PBSA and MM/ GBSA methods to estimate ligand-binding affinities. Expert Opin Drug Discov. 2015;10(5):449-461.

53. Wang C, Greene D, Xiao L, Qi R, Luo R. Recent developments and applications of the MMPBSA method. Front Mol Biosci. 2017;4:87.

54. Lees WD, Stejskal L, Moss DS, Shepherd AJ. Investigating substitutions in antibody-antigen complexes using molecular dynamics: a case study with broad-spectrum, influenza A antibodies. Front Immunol. 2017;8:143.

55. Wong S, Amaro RE, McCammon JA. MM-PBSA captures key role of intercalating water molecules at a protein-protein interface. JChem Theory Comput. 2009;5(2):422-429.

56. Wan S, Knapp B, Wright DW, Deane CM, Coveney PV. Rapid, precise, and reproducible prediction of peptide-MHC binding affinities from molecular dynamics that correlate well with experiment. JChem Theory Comput. 2015;11(7):3346-3356.

57. Wright DW, Hall BA, Kenway OA, Jha S, Coveney PV. Computing clinically relevant binding free 
energies of HIV-1 protease inhibitors. J Chem Theory Comput. 2014;10(3):1228-1241.

58. Jaigirdar A, Rosenberg SA, Parkhurst M. A High-avidity WT1-reactive T-cell receptor mediates recognition of peptide and processed antigen but not naturally occurring WT1-positive tumor cells. J Immunother. 2016;39(3):105-116.

59. Boudousquie C, Bossi G, Hurst JM, Rygiel KA, Jakobsen BK, Hassan NJ. Polyfunctional response by ImmTAC (IMCgp100) redirected $\mathrm{CD}^{+}$and $\mathrm{CD}^{+} \mathrm{T}$ cells. Immunology. 2017;152(3):425-438.

60. Park JH, Geyer MB, Brentjens RJ. CD19-targeted CAR T-cell therapeutics for hematologic malignancies: interpreting clinical outcomes to date. Blood. 2016;127(26):3312-3320.

61. Tikhonova AN, et al. $\alpha \beta$ T cell receptors that do not undergo major histocompatibility complex-specific thymic selection possess antibody-like recognition specificities. Immunity. 2012;36(1):79-91.

62. Van Laethem F, et al. Deletion of CD4 and CD8 coreceptors permits generation of alphabetaT cells that recognize antigens independently of the MHC. Immunity. 2007;27(5):735-750.

63. Feng D, Bond CJ, Ely LK, Maynard J, Garcia KC. Structural evidence for a germline-encoded $\mathrm{T}$ cell receptor-major histocompatibility complex interaction 'codon'. Nat Immunol. 2007;8(9):975-983.

64. Garcia KC, Adams JJ, Feng D, Ely LK. The molecular basis of TCR germline bias for MHC is surprisingly simple. Nat Immunol. 2009;10(2):143-147.

65. Scott-Browne JP, White J, Kappler JW, Gapin L, Marrack P. Germline-encoded amino acids in the alphabeta T-cell receptor control thymic selection. Nature. 2009;458(7241):1043-1046.

66. Dunbar J, Knapp B, Fuchs A, Shi J, Deane CM. Examining variable domain orientations in antigen receptors gives insight into TCR-like antibody design. PLoS Comput Biol. 2014;10(9):e1003852.

67. Cole DK, et al. T-cell receptor (TCR)-peptide specificity overrides affinity-enhancing TCR- major histocompatibility complex interactions. J Biol Chem. 2014;289(2):628-638.

68. Cole DK, et al. Increased peptide contacts govern high affinity binding of a modified TCR whilst maintaining a native $\mathrm{pMHC}$ docking mode. Front Immunol. 2013;4:168.

69. Madura F, et al. T-cell receptor specificity maintained by altered thermodynamics. JBiol Chem. 2013;288(26):18766-18775.

70. Aleksic M, et al. Dependence of T cell antigen recognition on T cell receptor-peptide $\mathrm{MHC}$ confinement time. Immunity. 2010;32(2):163-174.

71. Inaguma $Y$, et al. Construction and molecular characterization of a T-cell receptor-like antibody and CAR-T cells specific for minor histocompatibility antigen HA-1H. Gene Ther. 2014;21(6):575-584.

72. McKeithan TW. Kinetic proofreading in T-cell receptor signal transduction. Proc Natl Acad Sci U S A. 1995;92(11):5042-5046.

73. Tan MP, et al. T cell receptor binding affinity governs the functional profile of cancer-specific CD8+ T cells. Clin Exp Immunol. 2015;180(2):255-270.

74. van den Berg HA, et al. Cellular-level versus receptor-level response threshold hierarchies in T-cell activation. Front Immunol. 2013;4:250.

75. Stadinski BD, Obst R, Huseby ES. A "hot spot” for autoimmune $\mathrm{T}$ cells in type 1 diabetes. J Clin Invest. 2016;126(6):2040-2042.

76. Trenevska I, Li D, Banham AH. Therapeutic antibodies against intracellular tumor antigens. Front Immunol. 2017;8:1001.

77. Ahmed M, et al. TCR-mimic bispecific antibodies targeting LMP2A show potent activity against EBV malignancies. JCI Insight. 2018;3(4):97805.

78. Liu H, et al. Targeting alpha-fetoprotein (AFP)MHC complex with CAR T-cell therapy for liver cancer. Clin Cancer Res. 2017;23(2):478-488.

79. Harper J, et al. An approved in vitro approach to preclinical safety and efficacy evaluation of engineered $\mathrm{T}$ cell receptor anti-CD3 bispecific (ImmTAC) molecules. PLOS ONE. 2018;13(10):e0205491.
80. Cole DK, Dunn SM, Sami M, Boulter JM, Jakobsen BK, Sewell AK. T cell receptor engagement of peptide-major histocompatibility complex class I does not modify CD8 binding. Mol Immunol. 2008;45(9):2700-2709.

81. Barbas CF. Phage display: a laboratory manual. Cold Spring Harbor, New York, USA: Cold Spring Harbor Laboratory Press; 2001.

82. Turchaninova MA, et al. High-quality full-length immunoglobulin profiling with unique molecular barcoding. Nat Protoc. 2016;11(9):1599-1616.

83. Colaert N, Helsens K, Martens L, Vandekerckhove J, Gevaert K. Improved visualization of protein consensus sequences by iceLogo. Nat Methods. 2009;6(11):786-787.

84. Andreatta M, Lund O, Nielsen M. Simultaneous alignment and clustering of peptide data using a Gibbs sampling approach. Bioinformatics. 2013;29(1):8-14.

85. Case DA, Cerutti DS, Cheatham III TE, Darden TA, Duke RE, Giese TJ, et al. Amber 2016. University of California, San Francisco. 2016.

86. Chen VB, et al. MolProbity: all-atom structure validation for macromolecular crystallography. Acta Crystallogr D Biol Crystallogr. 2010;66(Pt 1):12-21.

87. Søndergaard CR, Olsson MH, Rostkowski M, Jensen JH. Improved treatment of ligands and coupling effects in empirical calculation and rationalization of pKa values. JChem Theory Comput. 2011;7(7):2284-2295.

88. Maier JA, Martinez C, Kasavajhala K, Wickstrom L, Hauser KE, Simmerling C. ff14SB: improving the accuracy of protein side chain and backbone parameters from ff99SB. JChem Theory Comput. 2015;11(8):3696-3713.

89. Jorgensen WL, Chandrasekhar J, Madura JD, Impey RW, Klein ML. Comparison of simple potential functions for simulating liquid water. JChem Phys. 1983;79(2):926-935.

90. Darden T, York DM, Pedersen LG. Particle mesh Ewald: An N·log $(\mathrm{N})$ method for Ewald sums in large systems. J Chem Phys. 1993;98(12):10089-10092. 